\title{
1 Interacting electrons in quasi-one-dimensional organic superconductors
}

\author{
C. Bourbonnais and D. Jérome \\ 1 Réseau Québecois sur les Matériaux de Pointe (RQMP), Département de \\ physique, Université de Sherbrooke, Sherbrooke, Québec, Canada J1K-2R1 \\ cbourbon@physique.usherb.ca \\ 2 Laboratoire de Physique des Solides, UMR 8502, Université de Paris-sud, 91405 \\ Orsay, France jerome@lps.u-psud.fr
}

\subsection{Introduction}

Superconductivity in organic materials has emerged in 1979 from an important background of preexisting knowledge and experimental techniques. All previous studies undertaken since 1973, which had been mostly performed on the (TTF - TCNQ) series of charge transfer organic conductors had failed to reveal superconductivity using chemistry and (or) pressure to suppress the density-wave or the so-called Peierls instability inherent to one-dimensional conductors. A breakthrough, which contributed to the discovery of organic superconductivity, has been the synthesis of the molecule TMTSF by K. Bechgaard and coworkers [1].

Actually, in the early 70's leading ideas governing the search for new materials likely to exhibit good metallicity and possibly superconductivity were driven by the possibility to minimize the role of electron-electron repulsions and at the same time to increase the electron-phonon interaction, while keeping the overlap between conducting stacks as large as possible. This led to the synthesis of the new electron donating molecule TMTSF, presenting much analogy with the previously known fulvalene donors in which the redox potential $(\Delta E)_{1 / 2}$ can be minimized $[2,3]$, by utilizing selenium instead of sulfur as hetero-atoms [4]. The next step was quite encouraging since the use of a high pressure has allowed to remove the instability due to the divergence of the Peierls channel down to the lowest temperatures in the two-chain conductor TMTSF - DMTCNQ [5]. A lucky situation has also been the synthesis of a series of $1 \mathrm{D}$ organic salts based on the radical cationic molecule TMTTF (the sulfur analog of the TMTSF molecule) and on a variety of inorganic monoanions such as $\mathrm{ClO}_{4}, \mathrm{BF}_{4}$ or SCN [6,7]. A conducting character could thus be anticipated from the intermolecular overlap of partly filled highest molecular orbitals (HOMO) of individual molecules. The compounds, $(\mathrm{TMTTF})_{2} \mathrm{X}$, were all insulating at ambient pressure but their crystal structure is the prototype of the (TMTSF $)_{2} \mathrm{X}$ series in which superconductivity has been subsequently discovered. In the rest of this article when we mention a $(\mathrm{TM})_{2} \mathrm{X}$ compound, this means that the organic molecule can be either TMTSF or TMTTF. The structure exhibits a face to 


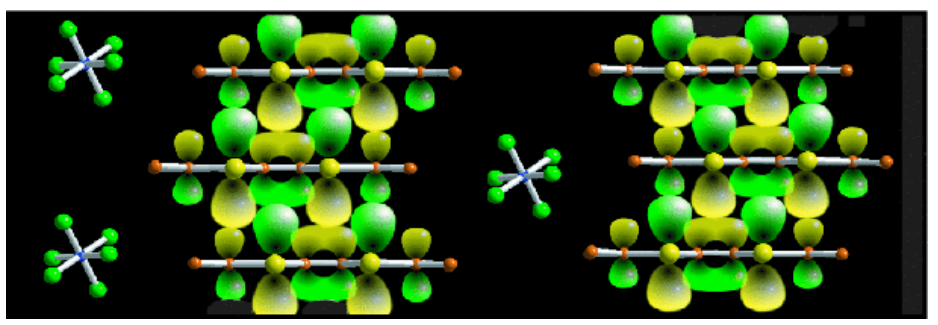

Fig. 1.1. $(\mathrm{TM})_{2} \mathrm{X}$, view of the cationic and anionic stacking perpendicular to the stacking axis, courtesy of J. Ch. Ricquier, IMN, Nantes

face packing of flat molecular units along the $a$ direction and the formation of molecular layers in the $a-b$ planes separated by anions stacks (Fig. 1.1). The overall symmetry is triclinic, not too far from orthorhombic often taken as the approximate structure by theoreticians. In addition, the structure reveals an important peculiarity namely, the anions are located in centrosymmetrical cavities lie slightly above or below each molecular plane with a zigzag stacking of the molecules along the $a$ direction. This structure leads in turn to a weak alternation of the interplanar distance (dimerization and a concomitant splitting of the HOMO conduction band into a filled lower sub-band separated from a half-filled upper (hole-like) band by a gap $\Delta_{D}$ at $\pm 2 k_{F}$, called the dimerization gap in the new Brillouin zone. However, on account of the finite transverse dispersion, this dimerization gap does not lead to a genuine gap in the middle of the density of states as given from the extended-Hückel band calculation. The only claim that can be made is that these conductors show commensurate band filling (three-quarter filled with electrons or one quarter-empty with holes). This originates from the 2:1 stoichiometry. Consequently, according to a non interacting particle band calculation, all compounds in the (TMTTF $)_{2} \mathrm{X}$ series should be found conducting.

Table 1.1. Calculated band parameters for three representative members of the $(\mathrm{TM})_{2} \mathrm{X}$ series according to the room temperature crystallographic data in Ref. [8]. The average intra and interstack interactions are given in lines 3 and 5 , respectively. The bond dimerization is shown in line 4 . All energies are in $\mathrm{meV}$

\begin{tabular}{lccc}
\hline & $(\mathrm{TMTTF})_{2} \mathrm{PF}_{6}(\mathrm{TMTSF})_{2} \mathrm{PF}_{6}(\mathrm{TMTSF})_{2} \mathrm{ClO}_{4}$ \\
\hline$t_{1}$ & 137 & 252 & 258 \\
$t_{2}$ & 93 & 209 & 221 \\
$\bar{t}$ & 115 & 230 & 239 \\
$\overline{\Delta t}_{\bar{t}}$ & 0.38 & 0.187 & 0.155 \\
$\bar{t}_{\perp b}$ & 13 & 58 & 44 \\
\hline
\end{tabular}


In Table (1.1) we report the band parameters of different members of the (TMTSF $)_{2} \mathrm{X}$ series as computed from crystallographic data [8]. The sulfur compounds exhibit bands that are significantly narrower and their crystallographic structure is more dimerized than those of the selenide compounds. $(\mathrm{TMTTF})_{2} \mathrm{Br}$ (not listed in Table (1.1)) is, however, an exception among the sulfur compounds with a dimerization of 0.13 , which is smaller than the value calculated for (TMTSF) ${ }_{2} \mathrm{ClO}_{4}$. This might be due to the calculation of electronic bands based on rather old and less accurate crystallographic data than those used for the other compounds [9]. All (TM) $)_{2} \mathrm{X}$ compounds with diversified anions can be gathered on a generic phase diagram displaying a wealth of different physical properties [10]. The gross features of the $(\mathrm{TM})_{2} \mathrm{X}$ phase diagram are shown in Figures 1.2 and 1.3. Compounds on the left hand side of the phase diagram, such as (TMTTF) ${ }_{2} \mathrm{PF}_{6}$, are insulators below room temperature with a broad metal to insulator transition, while those on the right hand side of (TMTTF $)_{2} \mathrm{Br}$ exhibit an extended temperature regime with a metallic behavior and a sharp transition towards an insulating ground state. Therefore, the cause of the insulating nature of some members of the (TMTTF $)_{2} \mathrm{X}$ series should be determined in relation to the role of e-e repulsion and low dimensionality as we shall show later. Although the most extensive pressure studies have been performed on (TMTSF $)_{2} \mathrm{PF}_{6}$ and (TMTTF $)_{2} \mathrm{PF}_{6}$, recent studies of other compounds of the (TMTTF $)_{2} \mathrm{X}$ series with $\mathrm{X}=\mathrm{ReO}_{4}, \mathrm{BF}_{4}$ and $\mathrm{Br}$ have shown that the main features observed under pressure in (TMTTF $)_{2} \mathrm{PF}_{6}$ or in $(\mathrm{TMTSF})_{2} \mathrm{PF}_{6}$ are also observed in other systems [11]. At this stage we may emphasize that the band filling of these materials is commensurate and in addition the existence of a dimerization in the crystal structure of the $(\mathrm{TM})_{2} \mathrm{X}$ series raises quite a challenging problem for the physics of one dimensional conductors since with the axial dimerization the conduction band becomes half-filled while it is originally quarter-filled from stoichiometry considerations. The commensurate band filling opens a new scattering channel for the carriers between both sides of the Fermi surface as then the total momentum transfer for two (four) electrons from one side of the 1-D Fermi surface to the other is equal to a reciprocal lattice vector (Umklapp scattering for half (quarter)-filled bands).

\subsection{Elements of theory for interacting electrons in low dimension}

In this section we shall depict some of the main results of the theory of quasione-dimensional metals. Given the pronounced one-dimensional anisotropy of the compounds, it is natural to first consider the 1D limit. To this end, the study of susceptibilities of non interacting electrons is particularly revealing of the natural instabilities that can take place in one dimension. Take for example the bare Peierls susceptibility of the system, which is the response to 


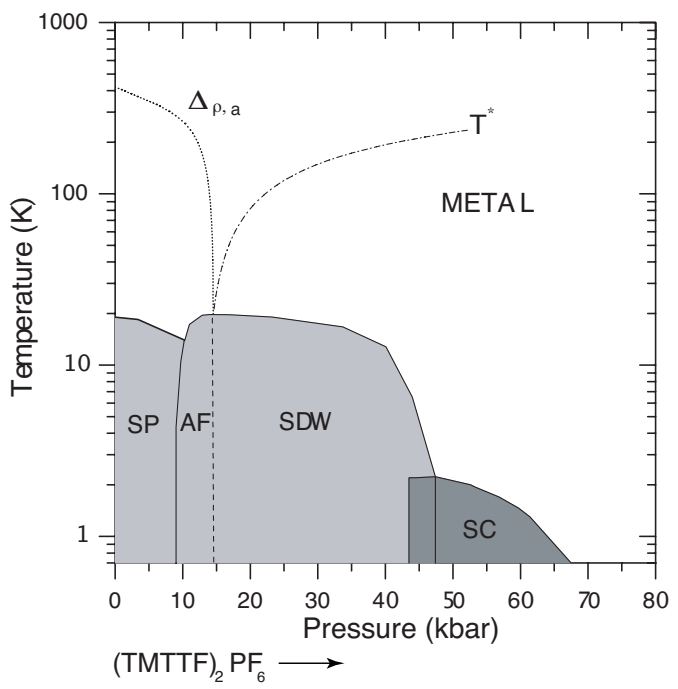

Fig. 1.2. Phase diagram of $(\mathrm{TMTTF})_{2} \mathrm{PF}_{6}$, as determined from transport measurements. After Ref. [11,12]

the formation of $2 k_{F}$ electron-hole pairs, the constituents of charge-densitywave $(\mathrm{CDW})$ and spin-density-wave (SDW) correlations (here $k_{F}$ being the Fermi wave vector). In one dimension, the energies of an electron (hole) state at $k$ and a hole (electron ) state at $k-2 k_{F}$ are connected through the nesting relation $\epsilon(k)=-\epsilon\left(k-2 k_{F}\right)$ of the electron spectrum $\epsilon(k)$ close to the Fermi level. The summation over a macroscopic number of intermediate electronhole states linked by this relation leads to an infrared logarithmic singularity of the form $\chi_{P}^{0}\left(2 k_{F}, T\right) \sim\left(\pi v_{F}\right)^{-1} \ln \left(E_{F} / T\right)$. Similarly, the Cooper susceptibility $\chi_{C}^{0}$, which probes the formation of pairs (two holes or two electrons) of particles of total momentum zero that are connected through the inversion property of the spectrum $\epsilon(k)=\epsilon(-k)$, also gives rise to a logarithmic divergence $\chi_{C}^{0}(T) \sim\left(\pi v_{F}\right)^{-1} \ln \left(E_{F} / T\right)$ - a singularity that is actually found in any dimension.

What thus really makes one dimension so peculiar resides in the fact that both singularities refer to the same set of electronic states and will then interfere one another [13]. In the presence of interactions, the interference is found to all order of perturbation theory for the scattering amplitudes of electrons with opposite Fermi velocities and it modifies the nature of the electron system in a essential way. In the framework of the 1D electron gas model, the selected emphasis put by these infrared singularities on electronic states close to the Fermi level allows us to define interactions with respect to the Fermi points $\pm k_{F}[14,15]$. Thus for a rotationally invariant system of length $L$, the Hamiltonian of the electron gas model can be written in the form 


$$
\begin{aligned}
H & =\sum_{k, p, \sigma} \epsilon_{p}(k) a_{p, k, \sigma}^{\dagger} a_{p, k, \sigma} \\
& +\frac{1}{L} \sum_{\{k, q, \sigma\}} g_{1} a_{+, k_{1}+2 k_{F}+q, \sigma}^{\dagger} a_{-, k_{2}-2 k_{F}-q, \sigma^{\prime}}^{\dagger} a_{+, k_{2}, \sigma^{\prime}} a_{-, k_{1}, \sigma} \\
& +\frac{1}{L} \sum_{\{k, q, \sigma\}} g_{2} a_{+, k_{1}+q, \sigma}^{\dagger} a_{-, k_{2}-q, \sigma^{\prime}}^{\dagger} a_{-, k_{2}, \sigma^{\prime}} a_{+, k_{1}, \sigma} \\
& +\frac{1}{2 L} \sum_{\{p, k, q, \sigma\}} g_{3} a_{p, k_{1}+p 2 k_{F}+q, \sigma}^{\dagger} a_{p, k_{2}-p 2 k_{F}-q+p G, \sigma^{\prime}}^{\dagger} a_{-p, k_{2}, \sigma^{\prime}} a_{-p, k_{1}, \sigma} \\
& +\frac{1}{L} \sum_{\{p, k, q, \sigma\}} g_{4} a_{p, k_{1}+q, \sigma}^{\dagger} a_{p, k_{2}-q,-\sigma}^{\dagger} a_{p, k_{2},-\sigma} a_{-p, k_{1}, \sigma}
\end{aligned}
$$

where $\epsilon_{p}(k)=v_{F}\left(p k-k_{F}\right)$ is the electron spectrum energy for right $(p=+)$ and left $(p=-)$ going electrons, $g_{1}$ and $g_{2}$ are the back and forward scattering amplitudes, respectively, whereas $g_{3}$ corresponds to the Umklapp scattering, a process made possible at half-filling, where the reciprocal lattice vector $G=4 k_{F}=2 \pi / a$ enters in the momentum conservation law; finally, one has the coupling $g_{4}$ by which two electrons near $k_{F}$ (resp. $-k_{F}$ ) experience a small momentum transfer which keeps them on the same branch [15].

In the one-loop perturbation theory, the electron scattering amplitudes are corrected by the aforementioned Cooper and Peierls logarithmic singularities. These logarithms are scale invariant quantities as a function of energy or temperature, which allow us to write down scaling or flow equations for the various scattering amplitudes. After all cancellations due to Cooper-Peierls interference being made, we have $[14,15]$,

$$
\begin{aligned}
& \tilde{g}_{1}^{\prime}=-\tilde{g}_{1}^{2}+\ldots \\
& \left(2 \tilde{g}_{2}^{\prime}-\tilde{g}_{1}^{\prime}\right)=\tilde{g}_{3}^{2}+\ldots \\
& \tilde{g}_{3}^{\prime}=\tilde{g}_{3}\left(2 \tilde{g}_{2}-\tilde{g}_{1}\right)+\ldots,
\end{aligned}
$$

where $\tilde{g}_{i}^{\prime}=(d / d \ell) \tilde{g}_{i}$ and $\ell=\ln E_{F} / T$ is the logarithmic - loop - variable. The long wavelength spin excitations are governed by the $\tilde{g}_{1} \equiv g_{1} / \pi v_{\sigma}$ $\left(v_{\sigma}=v_{F}\left(1-g_{4} / 2 \pi v_{F}\right)\right.$ coupling, which is decoupled from both $\tilde{g}_{3} \equiv g_{3} / \pi v_{\rho}$ $\left(v_{\rho}=v_{F}\left(1+g_{4} / 2 \pi v_{F}\right)\right)$ and the combination $2 \tilde{g}_{2}-\tilde{g}_{1} \equiv\left(2 g_{2}-g_{1}\right) / \pi v_{\rho}$ connected to charge excitations. In the physically relevant repulsive sector for systems like $(\mathrm{TM})_{2} \mathrm{X}$ where $g_{1,2}>0$, and owing to the existence of a small dimerization gap $\Delta_{D} \ll E_{F}$ of organic stacks (Table 1.1), weak half-filled Umklapp scattering $g_{3} \approx g_{1} \Delta_{D} / E_{F}$ is present $[16,17]$. Thus for $g_{1}-2 g_{2}<\left|g_{3}\right|$, both $2 g_{2}-g_{1}$ and $g_{3}$ are relevant variables for the charge and scale to the strong coupling sector, where a charge gap $\Delta_{\rho}$ is found below the temperature scale $T_{\rho}\left(\sim \Delta_{\rho} / 2\right)$. This can be seen as a $4 k_{F}$ charge localization responsible for a Mott insulating (MI) state. On the other hand, the solution $\tilde{g}_{1}(T)=\tilde{g}_{1} /\left(1+\tilde{g}_{1} \ln E_{F} / T\right)$ for the $g_{1}$ coupling, which follows from Eq. (1.2), is marginally irrelevant and scales to zero, leaving the spins degrees of freedom gapless as shown by the calculation of the uniform spin 
susceptibility $[14,18]$,

$$
\chi_{\sigma}(T)=\frac{2 \mu_{B}^{2}\left(\pi v_{\sigma}\right)^{-1}}{1-\frac{1}{2} \tilde{g}_{1}(T)} .
$$

The spin susceptibility decreases monotonically as a function of temperature and is unaffected by the occurrence of a charge gap. The electron system develops singularities, however, for staggered density-wave response. Thus the $2 k_{F}$ SDW or antiferromagnetic response, which is governed by the combination of couplings $\tilde{g}_{2}(\ell)+\tilde{g}_{3}(\ell)$ that flows to strong coupling, develops a power law singularity of the form

$$
\chi_{\mathrm{AF}}\left(2 k_{F}, T\right) \propto\left(\pi v_{F}\right)^{-1}\left(T / \Delta_{\rho}\right)^{-\gamma},
$$

where the power law exponent $\gamma=\tilde{g}_{2}\left(T_{\rho}\right)+\tilde{g}_{3}\left(T_{\rho}\right) \sim 1$. The response for the $2 k_{F}$ charge-density-wave 'on bonds', called the bond-order-wave (BOW) response, which is governed by the combination of couplings $\tilde{g}_{2}(\ell)+\tilde{g}_{3}(\ell)-$ $2 \tilde{g}_{1}(\ell)$, also develops a power law singularity in temperature

$$
\chi_{\mathrm{BOW}}\left(2 k_{F}, T\right) \propto\left(\pi v_{F}\right)^{-1}\left(T / \Delta_{\rho}\right)^{-\gamma_{\mathrm{BOW}}} .
$$

Here the exponent $\gamma_{\mathrm{BOW}} \sim 1$ is essentially the same as the one of AF response - the amplitude of the latter being larger, however [19]. When $2 k_{F}$ phonons are included, their coupling to singular BOW correlations yields a lattice instability of the spin-Peierls (SP) type. It is worthwhile to note that all the above properties of the $1 \mathrm{D}$ electron gas model find some echo in the phase diagram of $(\mathrm{TM})_{2} \mathrm{X}$ (Fig. 1.3).

\subsubsection{Some results of the bosonization picture [20]}

We now turn to the description of the one-dimensional electron gas using the bosonization method. A major property of interacting electrons in one dimension is that long wavelength charge or spin-density-wave oscillations constructed by the combination of electron-hole pair excitations at low energy form extremely stable excitations [20, 21]. Quasi-particles excitations like those taking place in a Fermi liquid (FL) for example, are absent at low energy and are replaced by these collective acoustic excitations for both spin $(\sigma)$ and charge $(\rho)$ degrees and freedom, thus allowing the construction of a phase representation of the electron gas Hamiltonian. The Fermi field

$$
\begin{gathered}
\psi_{p, \sigma}(x)=L^{-\frac{1}{2}} \sum_{k} a_{p, k, \sigma} e^{i k x} \\
\sim \lim _{\alpha_{0} \rightarrow 0} \frac{e^{i p k_{F} x}}{\sqrt{2 \pi \alpha_{0}}} \exp \left(-\frac{i}{\sqrt{2}}\left[p\left(\phi_{\rho}+\sigma \phi_{\sigma}\right)+\left(\theta_{\rho}+\sigma \theta_{\sigma}\right)\right]\right),
\end{gathered}
$$

can be expressed in terms of the spin and charge phase fields $\phi_{\nu=\rho, \sigma}[20,22]$ ( $\alpha_{0}$ is a short distance cut-off). These satisfy the commutation relations 


$$
\left[\Pi_{\nu^{\prime}}\left(x^{\prime}\right), \phi_{\nu}(x)\right]=-i \delta_{\nu \nu^{\prime}} \delta\left(x-x^{\prime}\right)
$$

where $\Pi_{\nu}(x)$ is the momentum conjugate to $\phi_{\nu}(x)$ and is defined by $\theta_{\nu}(x)=$ $\pi \int \Pi_{\nu}\left(x^{\prime}\right) d x^{\prime}$. In this phase variable representation the full electron gas Hamiltonian takes the form

$$
\begin{aligned}
H & =\sum_{\nu=\rho, \sigma} \frac{1}{2} \int\left[\pi u_{\nu} K_{\nu} \Pi_{\nu}^{2}+u_{\nu}\left(\pi K_{\nu}\right)^{-1}\left(\frac{\partial \phi_{\nu}}{\partial x}\right)^{2}\right] d x \\
& +\frac{2 g_{1}}{\left(2 \pi \alpha_{0}\right)^{2}} \int \cos \left(\sqrt{8} \phi_{\sigma}\right) d x+\frac{2 g_{3}}{\left(2 \pi \alpha_{0}\right)^{2}} \int \cos \left(\sqrt{8} \phi_{\rho}\right) d x .
\end{aligned}
$$

The harmonic part of the phase Hamiltonian on the first line corresponds to the Tomonaga-Luttinger model, which is exactly solvable. The spectrum shows no quasi-particles but only collective excitations and all the properties of the model then become entirely governed by the velocity $u_{\nu}$ and the 'stiffness constant' $K_{\nu}$ of acoustic excitations, which depend on interactions. This corresponds to the physics of the so-called Luttinger (LL) or TomonagaLuttinger liquid. In the Tomonaga-Luttinger limit the power law singularity of the AF spin response $\chi_{\mathrm{AF}}\left(2 k_{F}, T\right) \sim T^{-\gamma}$ is confirmed and the exponent

$$
\gamma=1-K_{\rho}
$$

is expressed in terms of the charge stiffness constant $K_{\rho}$. The absence of quasi-particle excitations is captured by the power law decay of the density of states at the Fermi level

$$
N(0) \sim\left(\pi v_{F}\right)^{-1}\left(\frac{T}{E_{F}}\right)^{\alpha},
$$

with the exponent

$$
\alpha=\frac{1}{4}\left(K_{\rho}+1 / K_{\rho}-2\right)
$$

The quasi-particle weigth at the Fermi level $z(T) \sim T^{\alpha}$ follows a similar power law decrease.

In the presence of the sine Gordon terms due to the backscattering and Umklapp couplings in the phase Hamiltonian Eq. (1.8), an exact solution cannot be found in the general case. However, a perturbative scaling procedure can be used for the various parameters that define the Hamiltonian [20]. For rotationally invariant repulsive couplings, $g_{1}$ is marginally irrelevant as found in the many-body description $(\S 1.2)$, and only the flow in the charge sector essentially matters. In low order, one can write the following flow equations

$$
\begin{aligned}
\frac{d K_{\rho}}{d l} & =-\frac{1}{2} K_{\rho}^{2} \tilde{g}_{3}^{2}, \\
\frac{d \tilde{g}_{3}}{d l} & =\tilde{g}_{3}\left(2-2 K_{\rho}\right) .
\end{aligned}
$$


For repulsive couplings, the bare $K_{\rho}=K_{\rho}\left(g_{4}, 2 g_{2}-g_{1}\right)<1, g_{3}$ is relevant and scales to strong coupling, as found in the previous fermion scaling description in Eqs. (1.2), while $K_{\rho}^{*}(l \gg 1) \rightarrow 0$. An expression for the charge gap can be found

$$
\Delta_{\rho} \sim W \tilde{g}_{U}^{1 /\left[2\left(1-n^{2} K_{\rho}\right)\right]},
$$

where for half-filling Umklapp $n=1$ and $\tilde{g}_{U}=\tilde{g}_{3}[20]$.

A charge gap is not limited to half-filling but may be present for other commensurabilities too [23]. At quarter-filling for example, the transfer of four particles from one side of the Fermi surface to the other leads to the Umklapp coupling

$$
H_{1 / 4} \simeq \frac{2 g_{1 / 4}}{\left(2 \pi \alpha_{0}\right)^{2}} \int d x \cos \left(2 \sqrt{8} \phi_{\rho}\right) .
$$

The phase argument of this term differs and leads to a distinct flow equation

$$
\frac{d \tilde{g}_{1 / 4}}{d l}=\left(2-8 K_{\rho}\right) \tilde{g}_{1 / 4},
$$

which goes to strong coupling if $K_{\rho}<1 / 4$, namely for sizable long-range Coulomb interaction [24]. The value of the insulating gap is given by (1.13) by taking $n=2$ and $\tilde{g}_{U}=\tilde{g}_{1 / 4}$ at quarter-filling $\left(\tilde{g}_{1 / 4} \sim(U / W)^{3}\right.$ in the Hubbard limit) $[24,25]$. It worth noting that in the special situation where the quarter-filled chains are weakly dimerized, both half-filling and quarter-filling Umklapp couplings are present in practice and should interfere one another [26]. In effect for materials like (TM) $)_{2} \mathrm{X}$, stoichiometry imposes half a carrier (hole) per TM molecule, a concentration that cannot be modified by applying pressure. Consequently, uniformly spaced molecules along the stacking axis should lead to a situation where a unit cell contains $1 / 2$ carriers, i.e., the conduction band is quarter-filled. However, the non-uniformity of molecular packing has been observed in early structural studies of the (TMTTF) ${ }_{2} \mathrm{X}$ crystals [8]. The dimerization of the overlap between molecules occurs along the stacks, a situation that is pronounced in the sulfur series, although it is also encountered in some members of the (TMTSF) $)_{2} \mathrm{X}$ series (Table 1). The impact of such a dimerization on the electronic structure is generally quantified by a modulation of the intra-stack overlap integral, because both longitudinal and transverse molecular displacements can contribute to the intermolecular overlap and could make them half-filled band compounds.

\subsubsection{The role of interchain coupling}

Electronic materials like $(\mathrm{TM})_{2} \mathrm{X}$ can be only considered as close realizations of $1 \mathrm{D}$ interacting fermion systems so that interchain coupling, though small, must be taken into account in their description. A non zero intermolecular overlap perpendicular to the chains yields finite interchain hopping integrals 


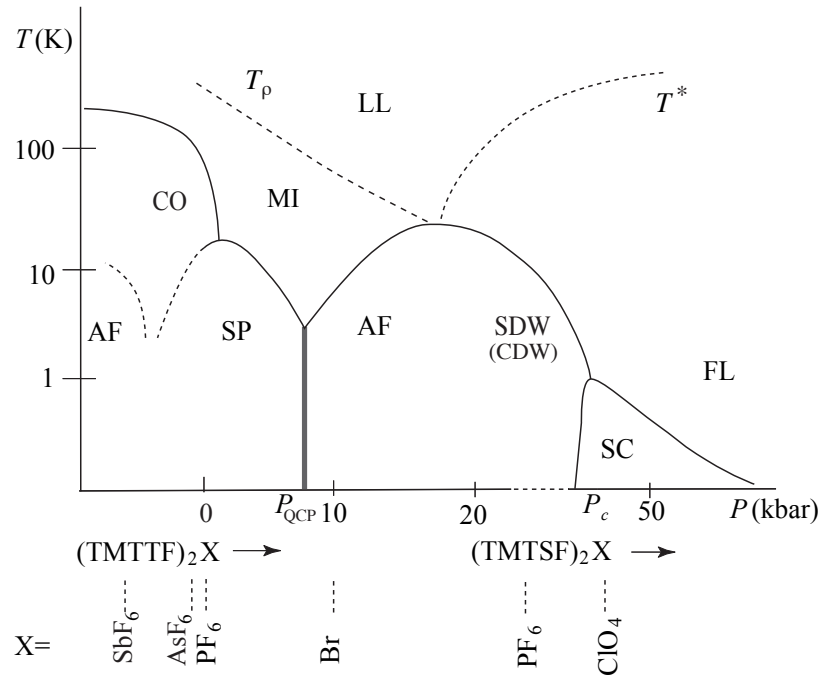

Fig. 1.3. The generic phase diagram of $(\mathrm{TM})_{2} \mathrm{X}$.

$t_{\perp b}$ and $t_{\perp c}$ along the $b$ and $c$ directions, respectively. These play an essential role either in the restoration of Fermi liquid (FL) quasi-particles or in establishing long-range order. Considering a square lattice of $N_{\perp}$ chains, the electron spectrum takes the form

$$
E_{p}(\mathbf{k})=\epsilon_{p}(k)-2 t_{\perp b} \cos k_{\perp b}-2 t_{\perp c} \cos k_{\perp c},
$$

where $\mathbf{k}=\left(k, k_{\perp b}, k_{\perp c}\right)$ and $t_{\perp c} \ll t_{\perp b} \ll E_{F}$. Owing to the strong anisotropy in the transfer integrals, a one-electron coherent motion in the transverse direction is not present at all temperatures. In the non interacting case for example, the temperature scale below which thermal fluctuations no longer blur the transverse quantum mechanical coherence for the electron is simply $T_{x^{1}} \sim t_{\perp b}$, which can be seen as a one-particle dimensionality crossover. In the presence of interactions, however, the quasi-particle weight $z(T) \sim\left(T / E_{F}\right)^{\alpha}$ being reduced by the LL behavior, the condition becomes $T_{x^{1}} \sim z\left(T_{x^{1}}\right) t_{\perp b}$ [27], namely

$$
T_{x^{1}} \sim t_{\perp b}\left(\frac{t_{\perp b}}{E_{F}}\right)^{\alpha / 1-\alpha} .
$$

The one-particle crossover scale $T_{x^{1}}$ then decreases in the presence of interactions. When a Mott insulating phase takes place in the 1D domain, $\alpha$ reaches unity, $T_{x^{1}}$ vanishes, and the transverse band motion is not possible the single-particle coherence becomes spatially confined along the stacks.

Transverse coherence is nevertheless possible but it is achieved through two-particle pair hopping, a mechanism for long-range order that is not present in the Hamiltonian at the start but which emerges when interactions along the stacks combine with $t_{\perp, b, c}$ in the one-dimensional region [28-31]. 
For repulsive interactions and commensurate fillings, the most important pair hopping processes that gradually emerges as a function of energy is an interchain antiferromagnetic exchange,

$$
\delta H_{\perp}=\sum_{i, j} \int d x J_{\perp i, j} \mathbf{S}_{i}(x) \cdot \mathbf{S}_{j}(x) .
$$

In the one-dimensional regime, it is governed at the one-loop level by the flow equation in the Fourier space

$$
\tilde{J}_{\perp}^{\prime}\left(\mathbf{q}_{0}\right)=\tilde{f}(\ell)+\tilde{J}_{\perp}\left(\mathbf{q}_{0}\right) \gamma(\ell)-\frac{1}{2}\left(\tilde{J}_{\perp}\left(\mathbf{q}_{0}\right)\right)^{2},
$$

where $\tilde{f}(\ell) \simeq-2 \sum_{i=b, c}\left[\left(\tilde{g}_{2}(\ell)+\tilde{g}_{3}(\ell)\right) t_{\perp, i} / W\right]^{2} e^{(2-2 \alpha(\ell)) \ell}$ and $\mathbf{q}_{0}=\left(2 k_{F}, \pi, \pi\right)$ is the modulation wave vector of AF order. Here $\alpha(\ell)$ and $\gamma(\ell)$ are scale dependent power law exponents of the quasi-particle weight (Eq. (1.11)) and antiferromagnetic susceptibility (Eq. (1.4)), respectively. Depending on the sign of $2-2 \alpha(\ell)-\gamma(\ell)$, different cases can be considered. Thus when a Mott gap is formed at $\ell_{\rho}=\ln E_{F} / T_{\rho}, 2-2 \alpha(\ell)-\gamma(\ell)$ becomes negative above $\ell_{\rho}$ and the solution of (1.18) can be put in the following simple Stoner form at temperature $T$

$$
\tilde{J}\left(\mathbf{q}_{0}\right) \approx \frac{\tilde{J}_{\perp b}+\tilde{J}_{\perp c}}{1-\left(J_{\perp b}+J_{\perp c}\right) \chi_{\mathrm{AF}}\left(2 k_{F}, T\right)}
$$

where the low temperature $1 \mathrm{D}$ AF susceptibility $\chi_{\mathrm{AF}}\left(2 k_{F}, T\right)$ is given by Eq. (1.4), for $\gamma=1\left(K_{\rho}^{*}=0\right)$, and

$$
J_{\perp b, c} \sim \pi v_{F} \frac{t_{\perp b, c}^{* 2}}{\Delta_{\rho}^{2}}
$$

is the effective exchange in the $b$ and $c$ directions at the scale $T_{\rho}$. From (1.19), a singularity will then occur at

$$
T_{c} \sim \frac{\left(t_{\perp b}^{* 2}+t_{\perp c}^{* 2}\right)}{\Delta_{\rho}},
$$

signaling a transition to a Néel ordered state. This result indicates that $T_{c}$ - essentially dominated by the exchange in the $b$ direction - increases as the Mott temperature $T_{\rho}$ decreases (Fig. 1.4). When the commensurability effects are decreasing and $T_{\rho}$ eventually merges with the critical behavior of the transition, antiferromagnetism becomes itinerant and corresponds to a SDW state. The interchain exchange enters in the weak coupling domain where $2-2 \alpha(\ell)-\gamma(\ell)$ is small but positive. This modifies the critical temperature, which reads

$$
T_{c} \sim \tilde{g}^{* 2} t_{\perp b}^{*},
$$

where $\tilde{g}^{*}=\tilde{g}_{2}^{*}+\tilde{g}_{3}^{*}$ and $t_{\perp b}^{*}=z t_{\perp b}$ are respectively the effective amplitude of electron-electron coupling and transverse hopping close to the transition. 


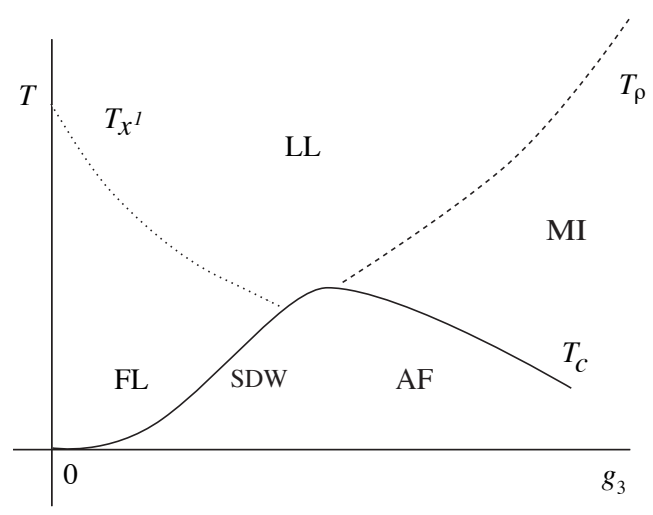

Fig. 1.4. Schematic phase diagram of a quasi-one-dimensional electron system at repulsive coupling calculated from the scaling theory. Here $g_{3} \propto g_{1,2}$ is the running Umklapp parameter for the amplitude of interaction.

The calculations show that $T_{c}$ starts to decrease in this domain giving rise to a maximum in $T_{c}[30,32,33]$. When the strength of $1 \mathrm{D}$ correlations becomes weaker one quickly arrives at the regime where $T_{x^{1}}$ becomes larger than the above $T_{c}$ so that the one-electron motion is no longer confined along the stacks and interchain coherence develops before the onset of criticality linked to the transition.

The temperature $T_{x^{1}}$ then becomes the scale below which the nesting of the whole Fermi surface becomes coherent. By looking at the effective spectrum Eq.(1.16) in which, $t_{\perp b, c} \rightarrow t_{\perp b, c}^{*}=z\left(T_{x^{1}}\right) t_{\perp b, c}$, perfect nesting occurs at $\mathbf{q}_{0}=\left(2 k_{F}, \pi, \pi\right)$ where the electron-hole symmetry $E_{-}^{*}(\mathbf{k})=-E_{+}^{*}\left(\mathbf{k}+\mathbf{q}_{0}\right)$ holds. It follows that electron-hole excitations within the energy shell $\sim T_{x^{1}}$ above and below the coherent - warped - Fermi surface lead to a logarithmically singular response $\chi^{0}\left(\mathbf{q}_{0}, T\right) \sim\left(\pi v_{F}\right)^{-1} \ln T_{x^{1}} / T$ in the Peierls channel. This singularity is also found in the perturbation theory of the scattering amplitudes and for repulsive interactions, it yields an instability of the normal state towards SDW long-range order. When perfect nesting prevails, a not too bad approximation consists of neglecting the interference between the Cooper and Peierls channels (we shall revert to the problem of interference below $T_{x^{1}}$ later in $\S$ 1.4.6). This corresponds to the ladder diagrammatic summation. In the scaling theory language, the ladder approximation corresponds to the flow equation

$$
\frac{d \tilde{J}}{d \ell}=\frac{1}{2} \tilde{J}^{2}+\ldots .
$$

for an effective coupling constant $\tilde{J}=\tilde{g}_{2}+\tilde{g}_{3}-\tilde{J}_{\perp}$ that defines the net attraction between an electron and a hole separated by $\mathbf{q}_{0}$. The integration 
leads to the Stoner expression

$$
\tilde{J}(T)=\frac{\tilde{J}^{*}}{1-\frac{1}{2} J^{*} \chi^{0}\left(\mathbf{q}_{0}, T\right)},
$$

where $\tilde{J}^{*}$ is the effective SDW coupling obtained from Eqs. (1.18) and (1.2) at $\ell_{x^{1}}=\ln E_{F} / T_{x^{1}}$, and $\chi^{0}\left(\mathbf{q}_{0}, T\right)=\left(\pi v_{F}\right)^{-1} \ln T_{x^{1}} / T$. The above expression leads to a BCS singularity at the SDW critical temperature

$$
T_{c}=T_{x^{1}} e^{-2 / J^{*}},
$$

which decreases as the interactions decrease. Nesting frustration is required to suppress the transition $[34,35]$. When nesting deviations are sufficiently strong, however, the FL remains unstable. Actually, when the partial but finite interference between the Peierls and the Cooper channels is restored, the system turns out to develop a superconducting instability. We shall return to this in $\S 1.4 .6$.

\subsection{The Fabre salts series}

\subsubsection{The generic $(\mathrm{TM})_{2} \mathrm{X}$ phase diagram}

Although the $(\mathrm{TM})_{2} \mathrm{X}$ generic phase diagram can be established by measuring the transport properties of various compounds, it has been most rewarding to use a single compound, namely (TMTTF ${ }_{2} \mathrm{PF}_{6}$, and the help of a high pressure to span the generic diagram in Figures 1.2 and 1.3. The study of this strongly insulating system (TMTTF) ${ }_{2} \mathrm{PF}_{6}$ under high pressure has been very useful not only because it has led to the stabilization of superconductivity in a strongly insulating sulfur compound [12], but also because its location at the left end of the phase diagram has allowed several key properties of quasi 1-D conductors to be carefully monitored under pressure. For instance, the longitudinal or transverse transports activation and the one-dimensional deconfinement arising under pressure [11], which will be discussed later in $\S 1.3 .3$. The phase diagram in Fig. 1.2 where $(\mathrm{TMTTF})_{2} \mathrm{PF}_{6}$ is the reference compound at ambient pressure has been obtained from the temperature dependences of $\rho_{c}(T)$ and $\rho_{a}(T)$ to be discussed below. In the low pressure region (see Fig. 1.5, $P<10 \mathrm{kbar}) \rho_{c}(T)$ and $\rho_{a}(T)$ are both activated, with $\Delta_{\rho, c}$ being about $30 \%$ larger than $\Delta_{\rho, a}$. At higher pressures, the activation of $\rho_{c}$ follows a gentle decrease, while $\Delta_{\rho, a}$ collapses abruptly at a pressure of about $14 \mathrm{kbar}$, which also marks the onset of a non-monotonous temperature dependence of $\rho_{c}$ with a maximum arising at the temperature denoted $T^{\star}$. Above $14 \mathrm{kbar}, \rho_{a}(T)$ displays a metallic temperature dependence down to the sharp metal-insulator transition below $20 \mathrm{~K}$, while $\rho_{c}(T)$ remains indicative of a weakly insulating state above the temperature $T^{\star}$, which increases 
under pressure. The different pressure dependences observed for longitudinal and transverse transport will be considered in the context of the pressureinduced deconfinement in $§ 1.3 .3$.

\subsubsection{Longitudinal transport}

A major problem encountered with the (TM) ${ }_{2} \mathrm{X}$ materials (as well as in most organic conductors) is the very strong pressure (or volume) dependence of their electronic properties, particularly in transport measurements [36-38]. This strong volume dependence is going along a particularly large thermal expansion. Hence, the only temperature dependence that can be compared with the prediction of the theory is the one measured at a constant volume. As all temperature dependences are obtained experimentally under constant pressure, a transformation to the constant volume $T$-dependence must be performed $[37,38]$, in order to make a significant comparison with the theory. Fortunately, this correction is not that relevant for the case of (TMTTF $)_{2} \mathrm{PF}_{6}$ under high pressure since the compressibility of these materials is known to decrease under pressure [39]. This is no longer the case when the metallic phase is already stable at ambient pressure as we shall see later for the case of (TMTSF $)_{2} \mathrm{PF}_{6}(\S 1.4 .1)$.

The insulating character of these materials with partly filled conduction bands is not expected in the framework of non interacting electrons. The reason for this must be the existence of strong repulsive interactions between 1D carriers. One explanation for the different values and pressure dependences of the activation energies can be taken as an evidence for in-chain conduction made possible by thermally excited 1-D objects similar to solitons in conducting polymers [40], whereas transverse transport requires the excitation of real quasi-particles (QP's) through a Mott-Hubbard gap larger than the soliton gap as we shall see. Contrasting with the strongly pressure dependence of the transport properties, the susceptibility is hardly sensitive to the location of a specific compound in the generic phase diagram. The spin susceptibility is dropping by about $40 \%$ between room and low temperature for all materials, although the actual magnitude and the low temperature behaviour depend on the compound (see Fig. 1.6). According to the 1D result Eq. 1.3 in the high temperature domain, a value of $\tilde{g}_{1} \sim 0.5$ can reasonably account for the temperature dependence of the magnetic susceptibility.

Although the insulating behavior of (TMTTF) ${ }_{2} \mathrm{X}$ salts can be clearly ascribed to their commensurate band filling, a closer examination is needed to determine which of the Umklapp scattering channels $1 / 2$ - or $1 / 4$-filled is the most active. In the presence of such a gap, the transport is activated at low temperature $\rho_{a} \propto e^{\Delta_{\rho, a} / T}$ but is expected to vary according to the power law

$$
\rho_{a} \propto T^{4 n^{2} K_{\rho}-3}
$$



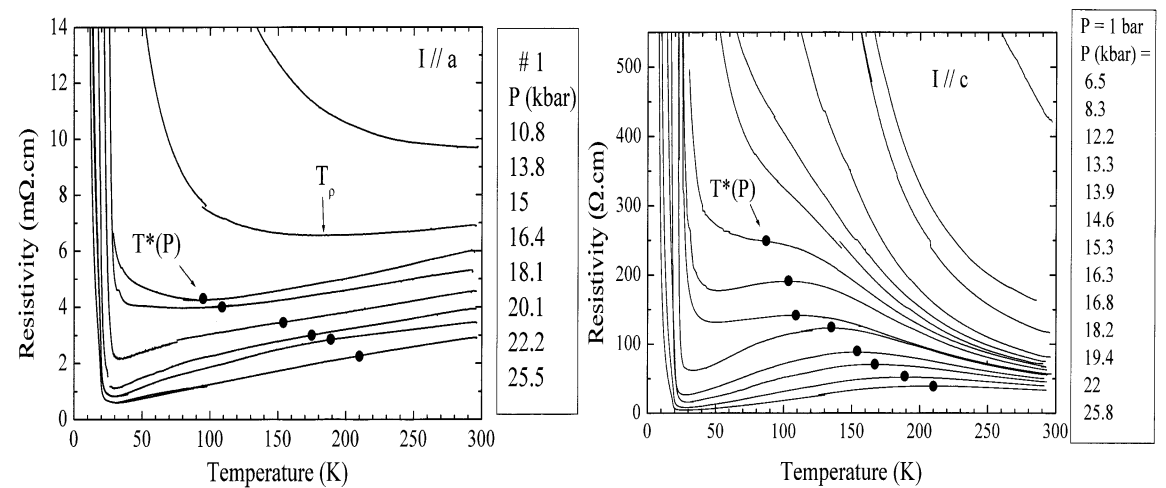

Fig. 1.5. (TMTTF $)_{2} \mathrm{PF}_{6}$, longitudinal (left) and transverse (right) resistances versus temperature at different pressures. After [11]

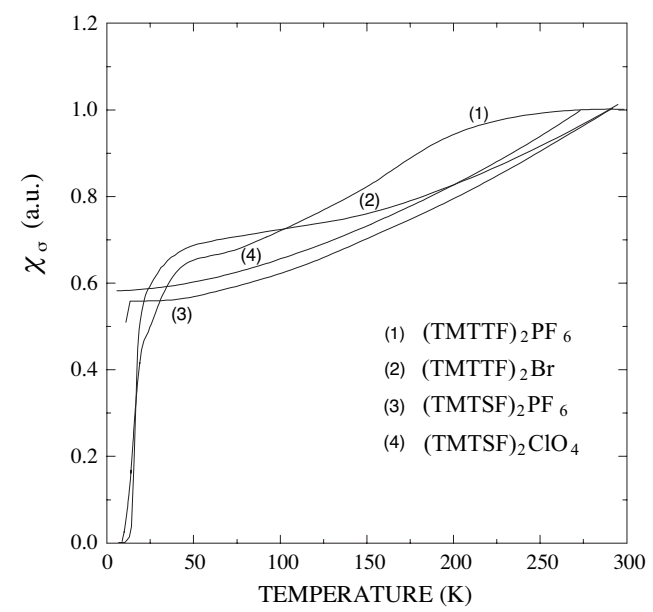

Fig. 1.6. Temperature dependence of the uniform spin susceptibility $\chi_{\sigma}$ of $(\mathrm{TM})_{2} \mathrm{X}$ compounds at ambient pressure. After References [41] [(TMTTF $\left.)_{2} \mathrm{PF}_{6}\right]$; [42] [(TMTTF $\left.)_{2} \mathrm{Br}\right] ;[43]\left[(\mathrm{TMTSF})_{2} \mathrm{PF}_{6}\right] ;[44]\left[(\mathrm{TMTSF})_{2} \mathrm{ClO}_{4}\right]$

in the high temperature regime, i.e., $T>\Delta_{\rho, a}$ [23]. The material resembles a metal at high temperature along the longitudinal direction. In the high $T$ 1-D regime $\left(T>t_{\perp b}\right)$, the picture of non-coupled chains is approached. Therefore, the density of quasiparticle states should resemble the situation that prevails in a Luttinger liquid namely, $N(\omega) \sim|\omega|^{\alpha}$, where $\alpha$ is related to a bare $K_{\rho}$ through Eq. (1.11), forgetting about the influence of the Mott gap (supposedly smaller than the temperature). 


\subsubsection{Transverse transport and deconfinement}

Under a pressure higher than 14 kbar the behavior of the $(\mathrm{TMTTF})_{2} \mathrm{PF}_{6}$ resistance along the direction of the weakest coupling, i.e., along the $c$-axis, displays an insulating character with a maximum around 80-100 K and becomes metallic at lower temperatures, although remaining several orders of magnitude above the Mott-Ioffe critical value, which is considered as the limit between metal and insulating-like transport [45]. Figure 1.7 displays the temperature dependence of $\rho_{c}$ in $(\mathrm{TMTTF})_{2} \mathrm{PF}_{6}$, and also for other members of the $(\mathrm{TM})_{2} \mathrm{X}$ family with different anions such as (TMTTF) ${ }_{2} \mathrm{BF}_{4}$ and $(\mathrm{TMTTF})_{2} \mathrm{Br}$.

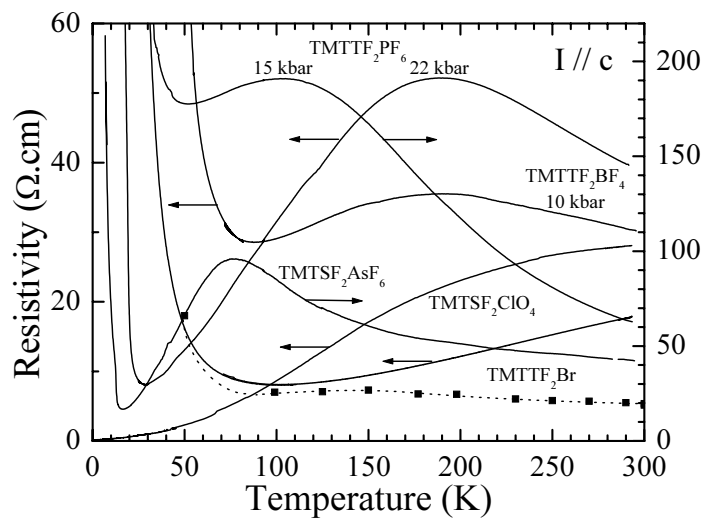

Fig. 1.7. Temperature dependence of the transverse transport along the c-axis in several compounds belonging to the $(\mathrm{TM})_{2} \mathrm{X}$ series studied under pressure. The temperature of the maximum of resistivity shows the location of the dimensional crossover $T^{\star}$, which is strongly pressure dependent for each compound. The resistivity upturn at low temperatures represents the stabilization of the insulating SDW phase. In case of the selenium compound (TMTSF) ${ }_{2} \mathrm{ClO}_{4}$, the crossover lies around room temperature whereas for the sulfur compound (TMTTF) ${ }_{2} \mathrm{Br}$ a resistivity maximum is seen only after the constant volume correction is taken into account. After Ref. [46]

The insulating character of the transverse transport has been interpreted as the signature of a non Fermi liquid behavior for carriers within planes (chains) [47]. When transverse transport along the $c$-direction is incoherent, transverse conductivity probes the physics of the $a-b$ planes and conductivity in terms of the transverse coupling $t_{\perp c}$ is expressed in the tunneling approximation as

$$
\sigma_{c}(\omega, T) \propto t_{\perp c}^{2} \int d x \int d \omega^{\prime} A_{1 D}\left(x, \omega^{\prime}\right) A_{1 D}\left(x, \omega+\omega^{\prime}\right) \frac{f\left(\omega^{\prime}\right)-f\left(\omega^{\prime}+\omega\right)}{\omega}
$$


where $A_{1 D}(x, \omega)$ is the one-electron spectral function of a single chain and $f(\omega)$ the Fermi-Dirac function. For $a-b$ planes made of an array of weakly interacting Luttinger chains, Eq. (1.27) leads to a power law temperature dependence for the $c$-axis conduction. The temperature at which the $c$-axis transport switches from an insulating to a metallic temperature dependence corresponds to a crossover between two regimes: a high temperature regime with no QP weight at the Fermi energy (possibly a TL liquid in the 1D case) and another regime in which the QP weight increases with decreasing temperature. This interpretation does not imply that the transport along the $c$-direction must also become coherent below the cross-over. The $c$-axis transport may well remain incoherent with a FL being established in the $a-b$ plane at temperatures below $T^{\star}$. The temperature dependence of the resistivity along the least conducting direction is thus expressed as [48]:

$$
\rho_{c}(T) \propto T^{1-2 \alpha} .
$$

Consequently, the temperature dependence of transport properties along the $a$ and $c$-axes above $T^{\star}$ should possibly lead to a consistent determination of $K_{\rho}$.

Now, regarding (TMTTF $)_{2} \mathrm{PF}_{6}$, we are facing a very interesting system, since the evolution from a Mott insulator to a metal can be carefully studied under pressure in a single sample and a decrease in compressibility under pressure makes constant volume correction less significant for temperature dependences measured at high pressures. Turning to the evaluation of the correlation coefficient from the temperature dependence of $\rho_{c}$, we end up fitting the data for (TMTTF) ${ }_{2} \mathrm{PF}_{6}$ in the pressure domain around $12 \mathrm{kbar}$, Fig. 1.5, with a very small value of $K_{\rho}$ (or large values of $\alpha$ ) which is not compatible with the value $K_{\rho}=0.23$ derived from the far infrared (FIR, see below) and NMR data [42]. Consequently, the Mott gap seems to be important in this temperature regime governing the excitation for the motion of single particles along $c$. Tentatively, one can expect a transverse resistivity behaving according to [48],

$$
\rho_{c}(T) \propto T^{1-2 \alpha} e^{\Delta_{\rho, c} / T} .
$$

Since the Mott -Hubbard gap varies as a power of $K_{\rho}$, even a small variation in the ratio between the Coulomb interaction and the bandwidth under pressure can explain a significant decrease of all gaps moving from the left to the right in the generic phase diagram. To summarize it is interesting to have a look at the data of longitudinal and transverse transports obtained in (TMTTF $)_{2} \mathrm{PF}_{6}$ under pressure displayed on Fig. 1.8. The transverse transport along $c$ is due to the hopping of quasi-particle and therefore requires an activation through a gap, which is the remanence of the Mott-Hubbard gap. It survives the onset of the dimensional cross-over. Thus we make the important identification 


$$
T^{\star} \equiv T_{x^{1}}
$$

between the temperature at which the metallic behavior in $\rho_{c}$ is restored and the single-particle dimensionality crossover Eq. (1.17). From Fig. 1.8, we see that $T^{\star}$ goes to zero when the insulating behavior in the longitudinal transport is restored, which is at $14 \mathrm{kbar}$ for a system like (TMTTF) ${ }_{2} \mathrm{PF}_{6}$, namely at the onset of electron confinement where the renormalization of $t_{\perp b, c}^{*}$ is strong (Eq. (1.17)). As for the longitudinal transport, it proceeds via the thermal excitation of 1-D objects similar to the solitons in conducting polymers through a gap smaller than the quasi-particle gap [40]. They loose their one-dimensional character and thus acquire a metallic power law temperature dependence when the transverse coupling becomes pertinent under pressure (i.e. above $14 \mathrm{kbar}$ ).

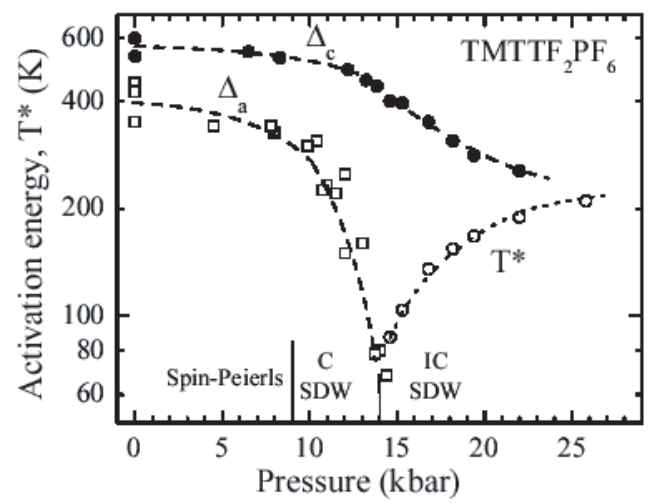

Fig. 1.8. Pressure dependence of the transport activation in (TMTTF) $)_{2} \mathrm{PF}_{6}$. The activation for the $c$ axis transport $\left(\Delta_{c} \equiv \Delta_{\rho, c}\right.$, in the text) although decreasing under pressure survives up to high pressures, while the longitudinal transport $\left(\Delta_{a} \equiv\right.$ $\Delta_{\rho, a}$, in the text) is no longer activated above $14 \mathrm{kbar}$ when the dimensional crossover arises at the finite temperature $T^{\star}$. After Ref. [11]

\subsubsection{Far infrared response in the $(\mathrm{TM})_{2} \mathrm{X}$ series}

An other signature of the Mott-Hubbard gap has been given by the frequencydependent conductivity $\sigma(\omega)$ measured in various salts of the $(\mathrm{TM})_{2} \mathrm{X}$ series exhibiting very different values of the conductivity at room temperature [49](see Fig. 1.9). The peak of the conductivity at a frequency $\omega_{0}$ correlates with the magnitude of the room temperature conductivity, namely both sulfur salts (TMTTF $)_{2} \mathrm{PF}_{6}$ and $(\mathrm{TMTTF})_{2} \mathrm{Br}$, which are insulating, exhibit a conductivity peak around $\omega_{0} \approx 1000 \mathrm{~cm}^{-1}$. In $(\mathrm{TMTSF})_{2} \mathrm{PF}_{6}$ the peak occurs around $200 \mathrm{~cm}^{-1}$ that is, very close to the zero frequency. Both 


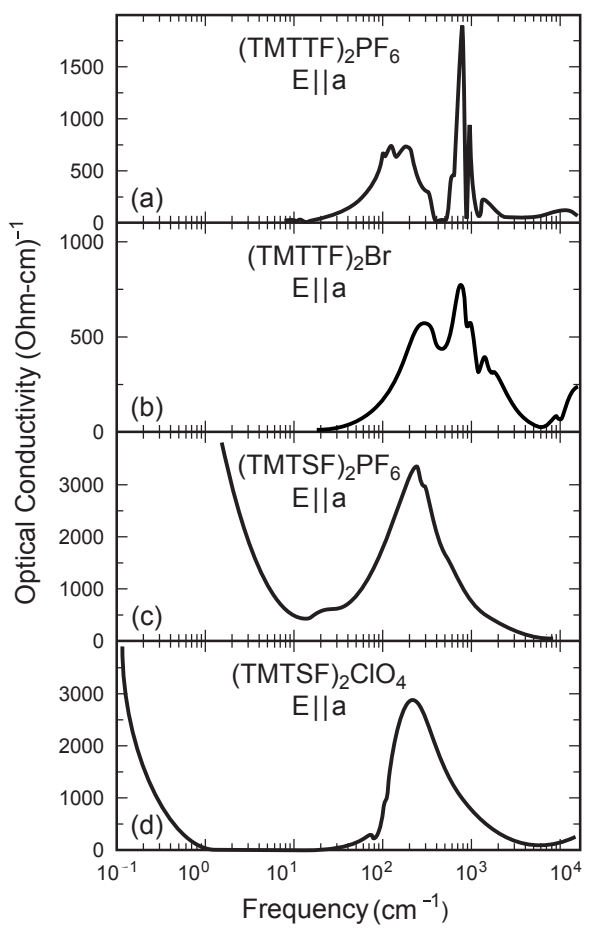

Fig. 1.9. Frequency dependence of the optical conductivity of several $(\mathrm{TM})_{2} \mathrm{X}$ compounds for the light polarized along the $a$ axis at ambient pressure and $T \approx 20 \mathrm{~K}$. After [50]

optical and transport data give $2 \Delta_{\rho, a}=800--1000 \mathrm{~K}$ in $(\mathrm{TMTTF})_{2} \mathrm{PF}_{6}$ at ambient pressure [11]. The difference between $K_{\rho}$ for selenium and sulfur compounds $\left(K_{\rho}=0.18\right.$ for the latter material) is a result of the difference between their bare bandwidths, since on-site repulsion, being a molecular property, is likely to be less sensitive to pressure than the intermolecular overlap along the stacking axis.

\section{Nuclear Magnetic Resonance}

The measurement of the temperature dependent nuclear spin-lattice relaxation rate in NMR denoted by $T_{1}^{-1}$ is another tool that has played a quite important role in the description of low energy electron spin correlations in $(\mathrm{TM})_{2} \mathrm{X}$ [42]. The connection between nuclear relaxation and the electron spin dynamics is given by the Moriya $T_{1}^{-1}$ expression

$$
T_{1}^{-1}=|A|^{2} T \int \frac{\chi^{\prime \prime}(\boldsymbol{q}, \omega)}{\omega} d^{D} q,
$$


which is taken in the zero Larmor frequency limit $(\omega \rightarrow 0)$ and where $A$ is proportional to the hyperfine matrix element. The relaxation of nuclear spins gives relevant information about the static, dynamics and dimensionality $D$ of electronic spin correlations. In $D=1$, this expression gives a relatively easy access to the interaction parameter $K_{\rho}$ that enters in most power laws expressions in one dimension $[18,27,51]$. According to Eqns. (1.3) and (1.4), the enhancement of the imaginary part of the spin susceptibility $\chi^{\prime \prime}$ occurs at $q \sim 0$ and $q \sim 2 k_{F}$, which yields

$$
T_{1}^{-1} \simeq C_{0} T \chi_{\sigma}^{2}(T)+C_{1} T^{K_{\rho}} .
$$

Here $C_{0}$ and $C_{1}$ contains weak logarithmic corrections in temperature. As a function of temperature, two different behaviors can be singled out. At high temperature, where uniform spin correlations dominate and those at $2 k_{F}$ are small, the relaxation rate is governed by the $T \chi_{\sigma}^{2}(T)$ term. In the low temperature domain, however, $2 k_{F}$ spin correlations are singularly enhanced, while uniform correlations remain finite so that $T_{1}^{-1} \sim T^{K_{\rho}}$.

Consider for example the insulating compounds (TMTTF $)_{2} \mathrm{X}$, we have seen in $\S 1.2 .1$ that the renormalized charge stiffness $K_{\rho}^{*} \rightarrow 0$ essentially vanishes below the Mott scale. The resulting behavior for the relaxation rate becomes

$$
T_{1}^{-1} \sim C_{1}+C_{0} T \chi_{\sigma}^{2} .
$$

As shown in Fig. 1.10, this behavior indeed emerges for (TMTTF) ${ }_{2} \mathrm{PF}_{6}$ salt when the relaxation rate is combined to the spin susceptibility data $\left(T \chi_{\sigma}^{2}\right)$ in the MI phase above three-dimensional ordering [51,52]. A similar linear behavior of $T_{1}^{-1}$ vs $T \chi_{\sigma}^{2}$, with a finite intercept confirming the $K_{\rho}^{*}=0$ value of the charge stiffness, is invariably found in all insulating materials down to the low temperature domain that surrounds the three-dimensional magnetic or lattice distorted long-range order $[37,42,53]$.

As one moves to the right-hand-side in the phase diagram of Fig. 1.3, we see that the $T_{1}^{-1}$ vs $T \chi_{\sigma}^{2}$ law is relatively well obeyed over a large temperature domain in the normal phase. If one takes (TMTSF) ${ }_{2} \mathrm{PF}_{6}$ for example, deviations are seen only below $150 \mathrm{~K}$ or so indicating that $K_{\rho}$ would be finite over the whole temperature range [42]. For (TMTSF) $)_{2} \mathrm{ClO}_{4}, T_{1}^{-1}$ enhancement coming from antiferromagnetic correlations emerges at even lower temperature $(\sim 30 \mathrm{~K})[27]$.

\subsubsection{The ordered states at low temperature}

\section{The spin-Peierls instability of the sulfur compounds}

The two compounds (TMTTF $)_{2} \mathrm{PF}_{6}$ and (TMTTF) ${ }_{2} \mathrm{AsF}_{6}$ of the Fabre series develop an instability of the MI phase that involves both spins and lattice degrees of freedom. X-ray diffuse scattering measurements of Pouget et al. [54], on both compounds have soon revealed the existence of diffuse 


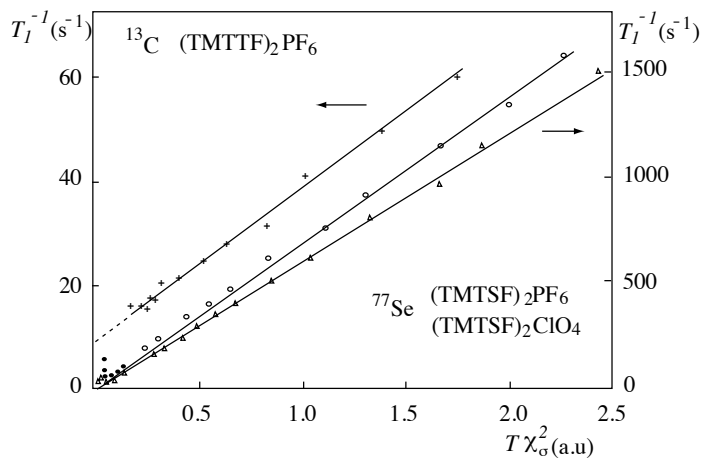

Fig. 1.10. Temperature dependence of NMR nuclear relaxation rate plotted as $T_{1}^{-1}$ vs $T \chi_{\sigma}^{2}$ for ${ }^{13} \mathrm{C}$ (TMTTF) ${ }_{2} \mathrm{PF}_{6}$ (crosses), ${ }^{77} \mathrm{Se}$ (TMTSF) ${ }_{2} \mathrm{PF}_{6}$ (open circles) and ${ }^{77} \mathrm{Se}$ (TMTSF) ${ }_{2} \mathrm{ClO}_{4}$ (open triangles). After Ref. [51]

scattering lines at the wave vector $2 k_{F}$ showing the onset of $1 \mathrm{D}$ lattice softening below the temperature scale $T_{S P}^{0} \approx 60 \mathrm{~K}$ for (TMTTF) ${ }_{2} \mathrm{PF}_{6}$ and $45 \mathrm{~K}$ for $(\mathrm{TMTTF})_{2} \mathrm{AsF}_{6}$. These lines condense into satellite reflections at the transition temperature $T_{S P} \approx 19 \mathrm{~K}$ for $(\mathrm{TMTTF})_{2} \mathrm{PF}_{6}$ salt and $15 \mathrm{~K}$, for (TMTTF $)_{2} \mathrm{AsF}_{6}$, where a static lattice distortion takes place at the wave vector $\mathbf{q}_{0}=\left(2 k_{F}, \pi, \pi\right)$. Recent elastic neutron scattering experiments did confirmed the existence of such a static distortion at $\mathbf{q}_{0}$ for $(\mathrm{TMTTF})_{2} \mathrm{PF}_{6}$ below $T_{\mathrm{SP}}$ [55]. Since both the softening and the transition occur in the Mott insulating state where only spin excitations are gapless, one thus deals with a spin-Peierls (SP) transition with a pronounced quasi-1D character. The expected non magnetic nature of both SP fluctuations and long-range order has been confirmed by the temperature dependence of the spin susceptibility (Fig. 1.6) and nuclear spin-lattice relaxation rate [42,56]; these quantities are reduced in the fluctuation regime and show thermal activation below $T_{\mathrm{SP}}$.

Following the example of the Peierls transition, the SP instability proceeds from the coupling of singular 1D bond-order-wave (BOW) electronic correlations to acoustic phonons at $2 k_{F}$ [57-59]. Unlike the Peierls case, however, the coupling becomes singular in the MI state instead of the metallic phase. The microscopic theory predicts a power law singularity in the 1D electronic BOW response below the Mott scale (Eq. 1.5), where for weakly dimerized chains systems like $(\mathrm{TMTTF})_{2} \mathrm{X}$, the power law exponent $\gamma_{\mathrm{BOW}}=1\left(K_{\rho}^{*}=0\right)$, as also found for the antiferromagnetic spin response Eq. (1.9). The enhancement of the electron-phonon interaction at $2 k_{F}$ by BOW correlations can be worked out by perturbation theory $[57,58]$. In the random phase approximation, the static temperature electron-electron vertex part induced by the exchange of $2 k_{F}$ phonons takes the form

$$
\Gamma_{\mathrm{ph}}\left(2 k_{F}, T\right)=\frac{g_{\mathrm{ph}}^{0}}{1-g_{\mathrm{ph}}^{0} \chi_{\mathrm{BOW}}\left(2 k_{F}, T\right)},
$$


where $g_{\mathrm{ph}}^{0}$ is the square of the bare $2 k_{F}$ electron-phonon matrix element. A singularity of the temperature vertex part will then develop at the mean field temperature

$$
T_{\mathrm{SP}}^{0}=c \tilde{g}_{\mathrm{ph}}^{0} T_{\rho},
$$

where $c \gtrsim 1$. Since there is no phase transition in one dimension, $T_{\mathrm{SP}}^{0}$ is not a true transition temperature but a temperature scale of lattice fluctuations that can be identified with the softening temperature seen in X-ray experiments. Although higher order fluctuations corrections will bring back the transition at $T=0, T_{S P}^{0}$ remains the right temperature scale for the onset of short-range fluctuations [57].

As for long-range SP order, it is driven by an interchain interaction which we denote $V_{\perp}$, whose contributions combine Coulomb, interchain hopping and three-dimensional phonons $[32,58]$. These coupling favor staggered bond order transversally to the chains at the wave vector $\mathbf{q}_{0}$. A molecular field treatment of interchain coupling, which takes into account one-dimensional fluctuations rigorously, leads to the mean-field expression for the spin-Peierls susceptibility

$$
\chi_{\mathrm{SP}}\left(\mathbf{q}_{0}, T\right)=\frac{\chi_{1 \mathrm{D}, \mathrm{SP}}\left(2 k_{F}, T\right)}{1-V_{\perp} \chi_{1 \mathrm{D}, \mathrm{SP}}\left(2 k_{F}, T\right)}
$$

where $\chi_{1 \mathrm{D}, \mathrm{SP}}$ is the $1 \mathrm{D}$ spin-Peierls fluctuations susceptibility. The singularity in $\chi_{\mathrm{SP}}$ occurs at

$$
T_{\mathrm{SP}}=T_{\mathrm{SP}}^{0} f\left(V_{\perp} / T_{\mathrm{SP}}^{0}\right),
$$

where from the singular behavior of $\chi_{1 \mathrm{D}, \mathrm{SP}}$ at low temperature [57], it is found that $f\left(V_{\perp} / T_{\mathrm{SP}}^{0}\right) \sim 1 / 3$ for $V_{\perp} / T_{\mathrm{SP}}^{0} \ll 1$ [58]. The resulting estimation $T_{\mathrm{SP}} \sim T_{\mathrm{SP}}^{0} / 3$ apparently holds for most electronically driven quasi-1D structural transitions in the adiabatic limit [60]. The observed values of the ratio $T_{\mathrm{SP}} / T_{\mathrm{SP}}^{0}$ in $(\mathrm{TMTTF})_{2} \mathrm{PF}_{6}$ and $(\mathrm{TMTTF})_{2} \mathrm{AsF}_{6}$ are compatible with this estimation.

The respective $T_{\mathrm{SP}}$ of $(\mathrm{TMTTF})_{2} \mathrm{AsF}_{6}$ and $(\mathrm{TMTTF})_{2} \mathrm{PF}_{6}$ evolve differently under low pressure. For (TMTTF $)_{2} \mathrm{AsF}_{6}, T_{\mathrm{SP}}$ first increases under pressure and reaches a maximum when the charge ordering temperature $T_{\mathrm{CO}}$ (see below) merges with $T_{\mathrm{SP}}$ at $1.5 \mathrm{kbar}$, and finally decrease at higher pressure [62]. Charge order breaks the inversion symmetry in the unit cell and acts as a $4 k_{F}$ charge-density-wave potential on molecular sites. The potential reduces the strength of BOW correlations [63], and in turn the amplitude of both $T_{\mathrm{SP}}^{0}$ and $T_{\mathrm{SP}}$. For $(\mathrm{TMTTF})_{2} \mathrm{PF}_{6}$, however, $T_{\mathrm{CO}}$ occurs at much lower temperature [64], and $T_{\mathrm{SP}}$ shows essentially a constant decrease under pressure. It finally goes down rapidly, extrapolating to zero at $P_{\mathrm{QCP}} \approx 9 \mathrm{kbar}$, where it competes with a Néel state $[52,61,65]$. This critical pressure corresponding to the zero temperature extrapolation of the critical lines can be put in the category of a quantum critical point (Fig. 1.11) [61]. 


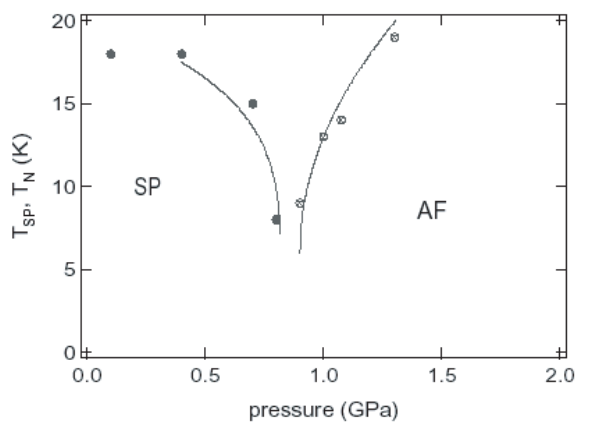

Fig. 1.11. The phase diagram of (TMTTF $)_{2} \mathrm{PF}_{6}$ near the quantum critical point at $P_{\mathrm{QCP}}$. After Ref. [61]

The decrease of $T_{\mathrm{SP}}^{0}$ and $T_{\mathrm{SP}}$ under pressure can be qualitatively understood if one considers that the drop in $T_{\rho}$ weakens electronic BOW correlations, which according to Eq. (1.35), reduces $T_{\mathrm{SP}}^{0}$ (Figs. 1.11 and 1.2). As $T_{\mathrm{SP}}^{0}$ carries on decreasing with pressure, it will reach values that become small compared to the typical energy $\omega_{D}$ of $2 k_{F}$ phonons $\left(\omega_{D} \sim 100 \mathrm{~K}\right.$ in these materials [66]), where quantum effects enter into play [67-69]. In effect, the above expression for $T_{\mathrm{SP}}^{0}$ has been obtained for 'static' phonons, in the so-called adiabatic approximation where the molecules are supposed to have an infinite mass. Adiabaticity is a reasonable assumption provided that $\omega_{D} / 2 \pi T_{\mathrm{SP}}^{0} \ll 1[67]$. As $T_{\mathrm{SP}}^{0}$ decreases and $\omega_{D}$ increases under pressure, however, the adiabatic condition will not be satisfied any more and the lattice softening will be reduced by the zero point motion of the molecular lattice. It has been shown that a quantum-classical crossover is expected at $\omega_{D} / \pi T_{\mathrm{SP}}^{0}(P) \approx 1$, where quantum corrections completely suppress $T_{\mathrm{SP}}^{0}$ and in turn $T_{\mathrm{SP}}[67,68]$. For a system like (TMTTF) ${ }_{2} \mathrm{PF}_{6}$, this would take place after a reduction of $T_{\rho}$ by a factor of two or so, which according to Fig. 1.3, corresponds to a pressure of the order of $P_{\mathrm{QCP}}$.

Charge ordering [70]

The MI state of most members of the Fabre series is characterized by another temperature scale connected to a different type of long-range order. The study of the temperature dependence of electrical permittivity for (TMTTF $)_{2} \mathrm{PF}_{6}$ and $(\mathrm{TMTTF})_{2} \mathrm{AsF}_{6}$ has indeed revealed the existence of a singularity in the dielectric constant at $70 \mathrm{~K}$ and $100 \mathrm{~K}$, respectively $[71,72]$. This singularity, not seen in the magnetic susceptibility (Fig. 1.6), is associated with an instability in the charge sector. The nature of this state was clarified at the same time by Chow et al. [64], who showed from NMR that the instability is actually a continuous phase transition towards a charge disproportionation in the unit cell. NMR does not tell, however, at which wave vector this charge ordered (CO) state takes place. In this regard, Monceau et al. [73], suggested that the 
anion lattice may undergo a uniform displacement when coupled to the $4 k_{F}$ electron charge instability along the stacks. In this picture, the CO instability in (TMTTF $)_{2} \mathrm{X}$ would be akin in most cases to a ferroelectric phase transition with a divergent dielectric constant.

The experimental identification of a charge-ordered state in (TMTTF $)_{2} \mathrm{X}$ salts lifted a sizable part of the veil surrounding the nature of the so-called 'structureless' phase transition that was detected much earlier from transport measurements for several members of the (TMTTF $)_{2} \mathrm{X}$ series $[74,75]$. It also puts an additional scale in the generic phase diagram of Fig. 1.3. Moreover, for a compound like (TMTTF) ${ }_{2} \mathrm{SbF}_{6}$ with a larger centro symmetrical anion, it was shown from NMR under pressure that the existence of both a CO transition at $T_{\mathrm{CO}} \simeq 150 \mathrm{~K}$ and a Néel state at $T_{c} \simeq 7 \mathrm{~K}$ forces to extend the pressure scale of Fig. 1.3, further on the left [76], where a Néel rather than a spin-Peierls state is stable. This Néel state is suppressed under pressure and replaced by a non magnetic phase, presumably of the spin-Peierls type such as found in (TMTTF $)_{2} \mathrm{PF}_{6}$ and (TMTTF $)_{2} \mathrm{AsF}_{6}$ [76], and which has been discussed above. At ambient pressure, (TMTTF) ${ }_{2} \mathrm{SbF}_{6}$ would then be found at the left of $(\mathrm{TMTTF})_{2} \mathrm{PF}_{6}$, which defines the origin on the pressure scale of Fig. 1.3. Other compounds like the $7 \mathrm{~K}$ antiferromagnet (TMTTF) ${ }_{2} \mathrm{SCN}$ would also be located in the same region of this extended phase diagram.

It is not clear, however, how far on the right-hand-side of Fig $1.3 \mathrm{CO}$ ordering is found. As mentioned above, it is known to be rapidly suppressed under pressure for compounds like (TMTTF) ${ }_{2} \mathrm{AsF}_{6}$ and (TMTTF) ${ }_{2} \mathrm{PF}_{6}$. However, it has been claimed to be present in compound like (TMTTF) $)_{2} \mathrm{Br}$ at ambient pressure [71].

The possibility for a $\mathrm{CO}$ order state, as a $4 k_{F}$ charge instability, is predicted to take place in purely quarter-filled one-dimensional system for small charge stiffness $K_{\rho}<1 / 4$ (Eq. (1.15)), namely for sizable long-range Coulomb interaction where it coincides with an insulating state [24,77]. It has been found also for models of interacting electrons in weakly dimerized chains with and without anions displacements $[26,78]$, in the framework of mean-field theory [79] and numerical calculations [80].

\section{The Néel order}

Sufficiently above $P_{\mathrm{QCP}}$ in Fig. 1.3, antiferromagnetic correlations within the MI state are much less affected by lattice SP fluctuations, which are sizably weaker in this pressure range. This is shown in the phase diagram by the absence of a spin pseudo gap from the temperature dependent nuclear relaxation rate in (TMTTF) $)_{2} \mathrm{PF}_{6}$ at $13 \mathrm{kbar}$ and in (TMTTF) ${ }_{2} \mathrm{Br}$ at 1 bar [42]. This is also confirmed in the case of (TMTTF $)_{2} \mathrm{Br}$ by X-ray diffuse scattering experiments at ambient pressure [81]. The observation in these conditions of a temperature independent nuclear relaxation rate for both materials, indicates that the power law exponent of the singularity in the $\mathrm{AF}$ response in Eq.(1.4) below the Mott scale $T_{\rho}$ is $\gamma=1\left(K_{\rho}^{*}=0\right)$ [42]. We have 
seen in $\S 1.2 .2$ that in the presence of a Mott gap, $\Delta_{\rho}>t_{\perp b}\left(\right.$ here $\left.\Delta_{\rho} \equiv \Delta_{\rho, a}\right)$, electron-hole bound pairs are formed and a coherent electron band motion in the transverse directions cannot take place. The propagation of order in the transverse directions leading to a Néel ordered state is provided by the antiferromagnetic interchain exchange $J_{\perp b, c}$ given by Eq. (1.20). We have seen that the temperature scale for the Néel ordering is determined by the singularity of the exchange coupling at the one-loop level (Eq. (1.18)), which leads to $T_{c} \propto 1 / \Delta_{\rho}$ (Eq. (1.21)).

This result indicates that $T_{c}$ - essentially dominated by the exchange in the $b$ direction - increases as the Mott gap $\Delta_{\rho}$ decreases, a feature commonly observed in the Fabre series (Figs. 1.3 and 1.2) $[82,83]$. It is worth noting that in the quarter-filled Mott insulator compound (EDT-TTF-CONMe $)_{2} \mathrm{AsF}_{6}$, the interchain exchange is also the driving force of antiferromagnetic longrange order. In these systems too, $T_{c}$ is found to increase as $\Delta_{\rho}$ is decreasing, a behavior that proved to be independent of the commensurability of Umklapp scattering processes behind the insulating gap (Fig. 1.4).

When the pressure is further increased, the Mott insulating and Néel critical scales meet and then the spins order themselves directly from the metallic state. Antiferromagnetism becomes itinerant in character and corresponds to a SDW state. The interchain exchange enters in the weak coupling sector and continues to be active, albeit on a relatively small pressure range with $T_{c}$ given by Eq (1.22). The calculations show that $T_{c}$ starts to decrease in this restricted pressure domain giving rise to a maximum in $T_{c}$ seen in experiments for (TMTTF $)_{2} \mathrm{Br}$ (Fig. 1.12) [82, 83], (TMTTF $)_{2} \mathrm{PF}_{6}$ [12] (Fig. 1.2) and mixed selenium-sulfur compounds (TMDMTSF) ${ }_{2} \mathrm{PF}_{6}[84,85]$. This weak coupling domain coupling quickly evolves to a regime where $t_{\perp b}^{*}$ and then the single electron transverse coherence length along the $b$ direction is increasing rapidly under pressure, signaling the beginning of a coherent band motion perpendicular to the chains. This yields the onset of electronic deconfinement and coherent nesting of the Fermi surface at $T^{\star}$ [86]. The value of $T_{c}$ in this latter domain also decreases when the couplings decrease under pressure (Fig. $1.4)$.

\subsection{The Bechgaard salts}

\subsubsection{The metallic phase}

The strongly metallic character of the (TMTSF $)_{2} \mathrm{PF}_{6}$ salt has been one of the highlights in the search for organic conductors [87]. The temperature dependence of the longitudinal resistivity follows a power law $T^{1.4}$ from $300 \mathrm{~K}$ down to about $100 \mathrm{~K}$. Below $35 \mathrm{~K}$, the resistivity of (TMTSF) ${ }_{2} \mathrm{PF}_{6}$ or (TMTSF $)_{2} \mathrm{AsF}_{6}$ is quadratic in temperature $\rho_{a}(T)=\rho_{0}+A T^{2}$, which is valid down to the metal-insulator transition due to the onset of an itinerant antiferromagnetic state at $12 \mathrm{~K}$ (Fig. 1.13). 


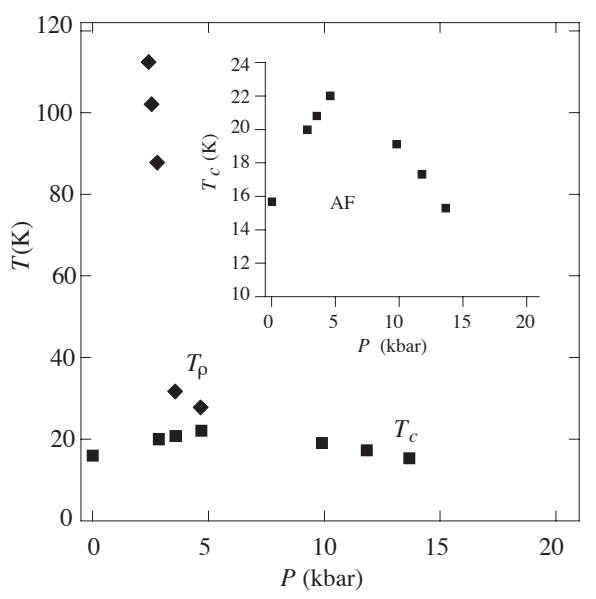

Fig. 1.12. Variation of the critical AF critical temperature as a function of hydrostatic pressure in (TMTTF $)_{2} \mathrm{Br}$. In the inset, a zoom of the maximum of $T_{c}$. After Ref. [82]

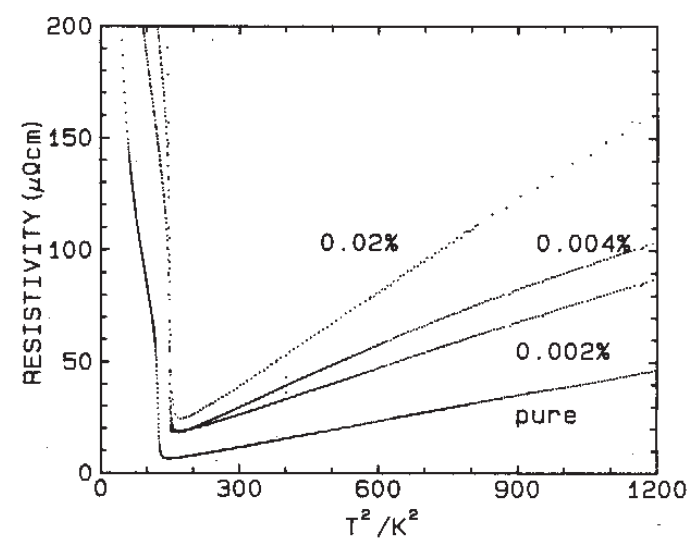

Fig. 1.13. Temperature dependence of the (TMTSF) ${ }_{2} \mathrm{PF}_{6}$ longitudinal resistivity plotted versus $T^{2}$ for pure and irradiated samples. After [88]

For high quality samples the resistance ratio $\rho_{a}(300 K) / \rho_{0}$ can reach values as large as 800 [88] (Fig.1.13). Furthermore, an interesting behaviour encountered in (TMTSF) ${ }_{2} \mathrm{PF}_{6}$ materials (and also in most organic conductors) is the very strong pressure (or volume) dependence of their electronic properties, particularly the transport property [36-38]. In addition, the thermal expansion of these materials is particularly large. Hence, the only temperature dependence that can be compared with the prediction of the theory is the one measured at a constant volume. As all temperature dependences are 
obtained under constant pressure, a constant volume transformation must be performed. An example is given in Fig. 1.14 by the longitudinal trans-

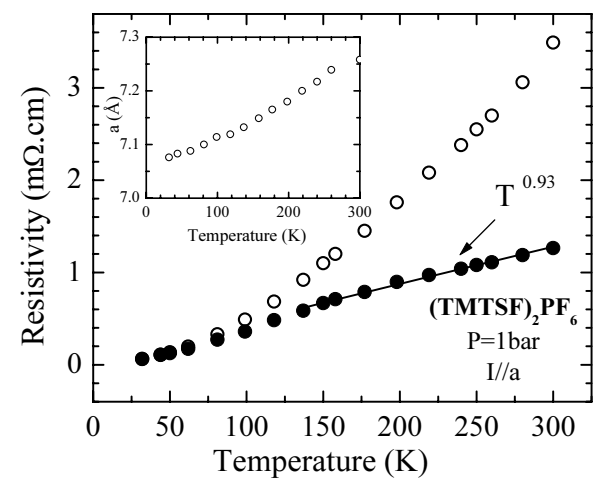

Fig. 1.14. Temperature dependence of the (TMTSF) ${ }_{2} \mathrm{PF}_{6}$ longitudinal resistivity at constant volume showing quasi linear $T$-dependence, with the thermal dependence of the lattice parameter $a$ displayed in the inset. After [38]

port of (TMTSF) $)_{2} \mathrm{PF}_{6}$ behaving at high temperature similar to $T^{2}$ under ambient pressure but varying sublinearly $\left(\sim T^{0.93}\right)$ from 300 to $150 \mathrm{~K}$, once the volume correction is taken into account [38]. The experimental power law of longitudinal resistivity leads in turn to $n^{2} K_{\rho}=0.98$ according to the theory of resistivity [23]. Note that a similar power law for the temperature dependence of longitudinal transport $\left(\sim T^{0.93}\right)$ can also be observed for two sulfur-compounds, $\mathrm{BF}_{4}$ and $\mathrm{PF}_{6}$ under high enough pressure when the correction to constant volume becomes negligible.

In the early days of the (TMTSF $)_{2} \mathrm{X}$ compound, the lattice dimerization was believed to govern entirely the amplitude of the Mott-Hubbard gap $[16,17]$. When the half-filled scenario is privileged, namely $(n=1)$. Hence, $n^{2} K_{\rho}=0.98$ leads to a bare value of $K_{\rho}$ close to unity implying a weakly coupled electron gas. This situation of a very weak coupling is difficult to reconcile with an enhancement of the spin susceptibility and the characteristic enhancement of the nuclear spin relaxation rate $[42,89]$ but an additional argument against this weak coupling is made possible by the unusual behavior of transverse transport (Eq. (1.28)) [48]. The weak coupling value for $K_{\rho} \approx 1$ derived from the temperature dependence of longitudinal transport and the optical data (see below) would imply $\alpha \approx 0$ and consequently a metal-like temperature dependence for $\rho_{c}(T)$ which is at variance with the data.

More recently, an alternative interpretation based on new experimental results has been proposed assuming that the $1 / 4$-filled scattering could justify the existence of the Mott gap in the entire $(\mathrm{TM})_{2} \mathrm{X}$ series [23]. With such a hypothesis $(n=2)$, the fit of the experimental data would thus lead to 
$K_{\rho}=0.23$ and $\alpha=0.64$ (see below, discussion on optical response) [90]. This bare value for $K_{\rho}$ agrees fairly well in the $1 / 4$-filled scenario at the very least at high temperature where the influence of half-filling Umklapp should be weak $[26,89]$. This value that would imply $U / W=0.7$ for the Hubbard parameter is compatible with plasma edge measurements and the enhancement in the spin susceptibility $[42,89]$. Such a strong coupling value for the bare $K_{\rho}$ implies that a system such as (TMTSF) ${ }_{2} \mathrm{PF}_{6}$ lies at the border between a 1D Mott insulator and a Luttinger liquid, though slightly on the insulating side. (TMTTF $)_{2} \mathrm{Br}$ is another particularly interesting system, in which the pressure coefficient of the resistivity is very large. Hence, it is the correction to constant volume which makes the maximum in $\rho_{c}$ to appear around $150 \mathrm{~K}$, while this maximum is absent in the constant pressure runs, (see Fig. 1.7).

The other approach to the correlation coefficient is given by the far infrared optical studies of (TMTSF $)_{2} \mathrm{PF}_{6}$, which have been very helpful for the determination of $K_{\rho}$ since the FIR gap of about $\Delta_{\rho, a}=200 \mathrm{~cm}^{-1}$ in (TMTSF) ${ }_{2} \mathrm{PF}_{6}$ has been attributed to the signature of the Mott-Hubbard gap [90]. Consequently, the frequency dependence of the conductivity above the Mott gap is closely linked to the dynamics of the excited carriers in the $1 \mathrm{D}$ regime. The theory predicts a power law dependence for the optical conductivity at frequencies larger than the Mott gap [23] namely,

$$
\sigma_{1, a}(\omega) \sim \omega^{4 n^{2} K_{\rho}-5}
$$

at $\omega>2 \Delta_{\rho, a}$ see Fig.(1.15).
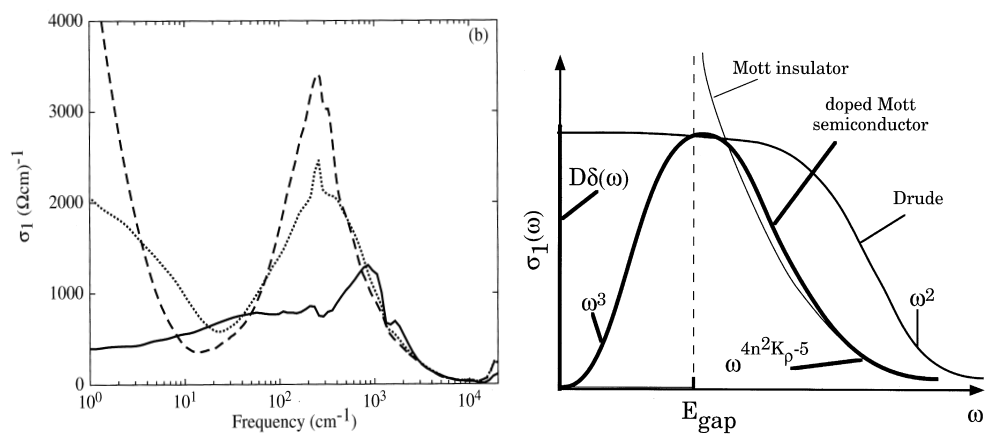

Fig. 1.15. Far infra red optical conductivity $(\mathbf{E} \| a)$ data of (TMTSF $)_{2} \mathrm{PF}_{6}$, experiment [90] for $T=300,100,20 \mathrm{~K}$ (left) and theory for a doped Mott insulator [23] (right)

According to the optical experimental data (see Fig.1.16 [90]), $\sigma_{1, a}(\omega) \propto$ $\omega^{-1.3}$ at high frequency leading to $n^{2} K_{\rho}=0.93$. This value for the correlation 


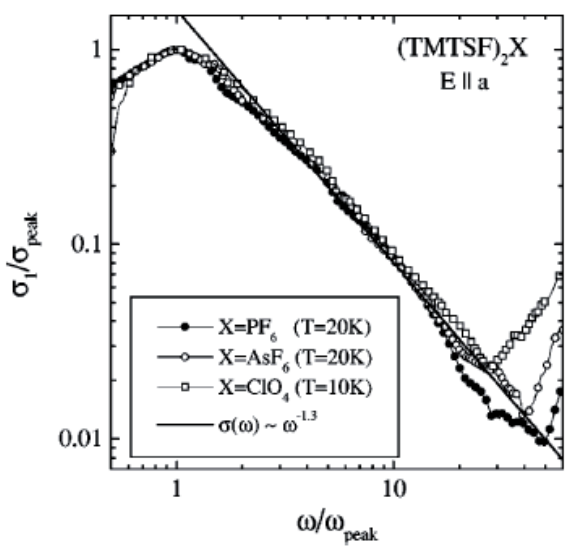

Fig. 1.16. Optical conductivity above the Mott-Hubbard gap in several selenide conductors analyzed in terms of a power law $\omega^{-1.3}$. After Ref. [90]

coefficient is fairly close to the one derived above from parallel transport data but none of these experiments allow by themselves to discriminate between half or quarter-filled Umklapp scattering.

\subsubsection{Pseudogap and zero frequency mode in the metallic phase of (TMTSF $)_{2} \mathrm{X}$}

It is also most illuminating to have a look at the conductivity in the far infrared regime. A large gap of order $1000 \mathrm{~K}$ is observed in the frequency dependence of the FIR conductivity of sulfur compounds [91]. This is in line with the activation energy of the DC conductivity in those compounds. However, the surprise arose for selenium compounds which behave apparently like normal metals as far as DC transport is concerned, in spite of the marked gap observed in the FIR regime at low temperature. The apparent normal behavior of the resistivity varying quadratically in temperature for (TMTSF $)_{2} \mathrm{ClO}_{4}$ or (TMTSF) ${ }_{2} \mathrm{PF}_{6}$ above the SDW transition could lead to the misleading conclusion of a 2 or 3-D Fermi gas in which the temperature dependence of the transport is governed by e-e scattering. However, the analysis of the conductivity in terms of the frequency reveals quite a striking breakdown of the Drude theory for single-particles. The inability of the Drude theory to describe the optical conductivity has been noticed by a number of experimentalists working on (TMTTF) $)_{2} \mathrm{X}$ with $\mathrm{X}=\mathrm{ClO}_{4}, \mathrm{PF}_{6}$ or $\mathrm{SbF}_{6}$ [92]. When the reflectance of (TMTSF) ${ }_{2} \mathrm{ClO}_{4}$ in the near-infrared is analyzed with the Drude model in the whole range of temperatures from 300 down to $30 \mathrm{~K}$ the electron scattering rate is found to decrease gradually from $2.5 \times 10^{14} \mathrm{~s}^{-1}$ at room temperature to $1.3 \times 10^{14} \mathrm{~s}^{-1}$ at $30 \mathrm{~K}$ [93]. Even if the RT value is not far from the value from DC conductivity, a drastic difference emerges 
at low temperature as $\sigma_{\mathrm{DC}}$ increases by a factor about 100 between $\mathrm{RT}$ and $30 \mathrm{~K}$ [94], as compared to the factor 2 for the optical lifetime.

An other striking feature of the optical conductivity has been noticed when the Kramers-Krönig transformation of the reflectance is performed in a broad frequency domain for (TMTSF $)_{2} \mathrm{ClO}_{4}$ as well for all conducting materials at low temperature. Given the usual Drude relation $\sigma_{\mathrm{DC}}=\omega_{p}^{2} \tau / 4 \pi$ between transport lifetime and plasma frequency data (the plasma frequency has been found nearly temperature independent $[49,93])$ and the measured resistance ratio for $\rho_{a}$ of about 800 between RT and $2 \mathrm{~K}$ obtained in good quality measurements, the Drude conductivity in the frequency range $\approx 40 \mathrm{~cm}^{-1}$ should amount to at least $4000 \Omega \mathrm{cm}^{-1}[95,96]$. The measured optical conductivity is at most of the order of $500 \Omega \mathrm{cm}^{-1}$ [95]. Consequently, the rise in the conductivity as $\omega \rightarrow 0$ has been taken in (TMTSF) ${ }_{2} \mathrm{ClO}_{4}$ as well as in the other salts with $\mathrm{PF}_{6}$ or $\mathrm{SbF}_{6}$ as an evidence for a hidden zero frequency mode. This mode is actually so narrow that it escapes a direct determination from K-K analysis of the reflectance, which is limited to the frequency domain above $10 \mathrm{~cm}^{-1}$. Estimates of the mode width have been obtained using the DC conductivity and the oscillator strength $\Omega_{p}^{2}$ of the mode with $\sigma_{\mathrm{DC}}=\Omega_{p}^{2} \tau / 4 \pi$ where $\Omega_{p}$ is measured from the first zero crossing of the dielectric constant. This procedure gives a damping factor $\Gamma=0.005 \mathrm{~cm}^{-1}$ and $0.09 \mathrm{~cm}^{-1}$ at 2 and $25 \mathrm{~K}$ respectively, in (TMTSF) ${ }_{2} \mathrm{ClO}_{4}$ [96]. The con-

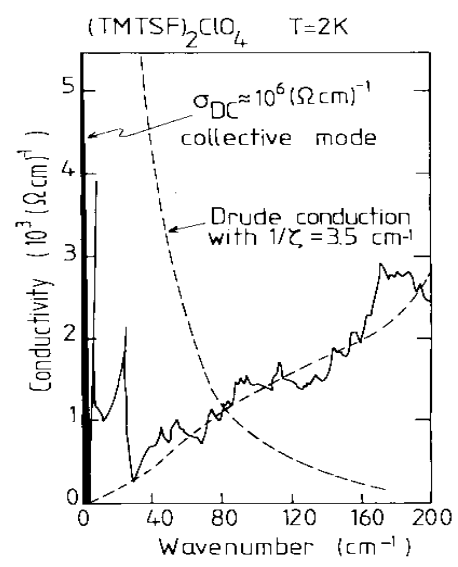

Fig. 1.17. Far infra red data of (TMTSF $)_{2} \mathrm{ClO}_{4}$. The dashed line is the Drude behaviour with $1 / \tau=3.5 \mathrm{~cm}^{-1}$ and $\omega_{p}=10^{4} \mathrm{~cm}^{-1}$. After Ref. [96]

firmation of a very long scattering time for the DC conduction has also been brought by the rapid suppression of $T_{c}$ by non-magnetic defects in the non conventional superconductor (TMTSF) ${ }_{2} \mathrm{ClO}_{4}$ leading to $\Gamma_{c}=0.56 \mathrm{~cm}^{-1}$ at 
low temperature [97], meaning that the electron lifetime at low temperature is actually much longer than the value inferred from a Drude description.

There is now a wealth of experimental evidences showing the development of a narrow frequency mode in the Mott gap of (TMTSF) ${ }_{2} \mathrm{ClO}_{4}$ and related conducting compounds. From FIR data in (TMTSF) ${ }_{2} \mathrm{PF}_{6}$, it has also been shown that the narrow mode carries only a small fraction (a few percent) of the total spectral weight $[49,90]$, but it is this mode that explains the very large value of the DC conduction observed at low temperature.

\subsubsection{Quarter-filled compounds}

Even if the debate between $1 / 2$ and $1 / 4$ fillings may be relevant for $(\mathrm{TM})_{2} \mathrm{X}$, this is no longer the case for new synthesized compounds in a family whose general structure precludes any dimerization. The structural peculiarity of the salt $\left(\mathrm{EDT}-\mathrm{TTF}-\mathrm{CONMe}_{2}\right)_{2} \mathrm{AsF}_{6}$ is the absence of inversion center between adjacent molecules in stacks and instead the presence of a glide symmetry plane [98] (see Fig.1.18). The analysis of the transport data of $(\mathrm{EDT}-\mathrm{TTF}-\mathrm{CONMe})_{2} \mathrm{AsF}_{6}$ has shown that in spite of the existence of a glide symmetry plane, the carriers are localized, and even more localized than in the most insulating salts of the Fabre series known at present. Since the localization in this compound cannot be ascribed to a $1 / 2$-Umklapp scattering or to the Anderson localization, 1/4-Umklapp scattering seems to be the only channel left to explain carrier localization in this commensurate 1D conductor. Under ambient pressure, given the total bandwidth deduced from quantum chemistry $\left(W=W_{\text {tot }}(P=1\right.$ bar $\left.)=0.350 \mathrm{eV}(3850 \mathrm{~K})\right)$ and the experimental Mott gap $\left(2 \Delta_{\rho, a}=2700 \mathrm{~K}\right)$, the theory [23] gives, in the case of quarter filling stricto sensu: $2 \Delta_{\rho, a}=2 W(U / W)^{3 / 2\left(1-4 K_{\rho}\right)}$. This leads to the bare value $K_{\rho}=0.1$, with a reasonable $U / W=0.7$.

The Mott gap of $\left(\mathrm{EDT}-\mathrm{TTF}-\mathrm{CONMe}_{2}\right)_{2} \mathrm{AsF}_{6}$ is much larger than the expected value of the bare interstack overlap $t_{\perp}$, which makes according to Eq. 1.17 the single particle hopping between neighboring stacks non-pertinent in the pressure regime less than $20 \mathrm{kbar}$ since the transverse hopping is renormalized to zero on account of a strong intrachain electron-hole interaction.

The interaction over bandwidth ratio $U / W \approx 0.7$ is also in fair agreement with the result of a crude analysis of the spin susceptibility of S-salts $[42,89]$ and indicates that these compounds lie in the strong coupling sector. With increasing pressure, the gap decreases steadily up to the pressure of $20 \mathrm{kbar}$ above which it disappears sharply due to the competing transverse coupling. Below $15 \mathrm{kbar}$, the gap of $\left(\mathrm{EDT}-\mathrm{TTF}-\mathrm{CONMe}_{2}\right)_{2} \mathrm{AsF}_{6}(1350 \mathrm{~K})$ is about equal to the gap of (TMTTF $)_{2} \mathrm{X}$ measured under ambient pressure [47]. Since the logarithmic pressure dependences of the gap seem to be similar for both compounds, we may say that $\left(\mathrm{EDT}-\mathrm{TTF}-\mathrm{CONMe}_{2}\right)_{2} \mathrm{AsF}_{6}$ can also be considered as part of the generic Fabre-Bechgaard salt diagram, provided the origin of the pressure axis is shifted to the left by $15 \mathrm{kbar}$. Hence, it is reasonable to expect that the TL parameter $K_{\rho}$ increases from left to right in 
the $(\mathrm{TM})_{2} \mathrm{X}$ diagram, since both optical and transport data suggest $K_{\rho}=0.23$ in the Se compounds whilst it is only of the order of 0.1 in sulfur compounds. Let us add that the uniformly stacked 1-D conductor (DI - DCNQI $)_{2} \mathrm{Ag}$, an

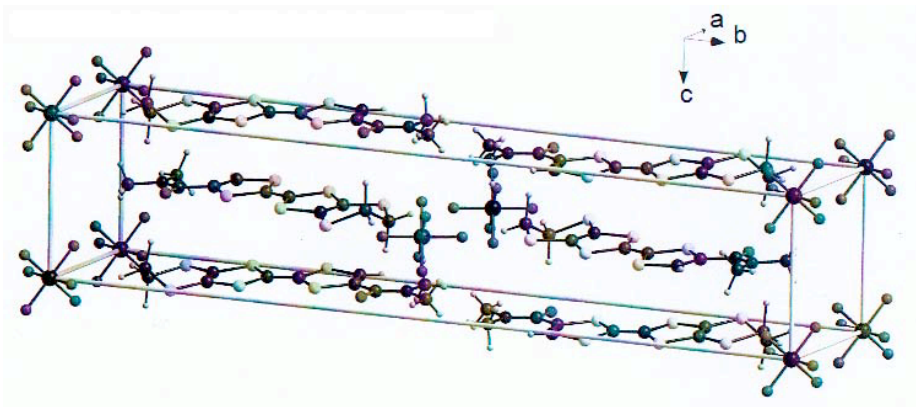

Fig. 1.18. Structure of the quarter-filled compound $\left(\mathrm{EDT}-\mathrm{TTF}-\mathrm{CONMe}_{2}\right)_{2} \mathrm{AsF}_{6}$. After Ref. [98]

other quarter-filled compound reveals localization properties quite similar to those observed in the $(\mathrm{TM})_{2} \mathrm{X}$ series [99]. The normal state of this system is insulating at low pressure probably due to strong electron correlations but at pressures exceeding $15 \mathrm{kbar}$, the longitudinal restivity is metallic above $100 \mathrm{~K}$ with a quasi linear temperature dependence leading to $K_{\rho}=0.25$ in the quarter-filled band hypothesis. This result shows once more that the conductor lies at the border with the quarter-filled Mott localized insulator.

\subsubsection{A robust 1-D compound:(TTDM-TTF $)_{2} \mathrm{Au}(\mathrm{mnt})_{2}$}

For the sake of completeness we shall mention the behavior of an interesting organic salt, which unlike all $(\mathrm{TM})_{2} \mathrm{X}$ compounds, has failed to reveal the usual suppression of the insulating phase under pressure [100]. The (TTDM$\mathrm{TTF})_{2} \mathrm{Au}(\mathrm{mnt})_{2}$ system, in spite of a strong structural analogy with the $(\mathrm{TM})_{2} \mathrm{X}$ materials exhibiting stacks of donors arranged in layers with short interchain contacts, shows a unique extreme 1-D character together with a strong bond dimerization. According to extended Huckel calculations, the dimerization gap at the middle of the HOMO band amounts to $0.027 \mathrm{eV}$ i.e $13 \%$ of the upper band dispersion and the interchain coupling within the layers is practically zero. As the smallness of this contact is due to terminal sulfur atoms in intermolecular contacts not participating to the HOMO of the molecule, we can expect a survival of the 1-D character under pressure and no pressure-induced dimensionality crossover. Consequently, contrasting with the members of the Fabre-Bechgaard series, the Mott-Hubbard insulating nature persists up to a pressure of $25 \mathrm{kbar}$ [100], which is usually large enough 
to severely decrease (if not suppress) the localization in the latter family as shown in the present article.

\subsubsection{The spin-density-wave phase}

At the core of the unity shown by the phase diagram of Fig. 1.3 is the shift on the pressure scale when selenium is substituted for the sulfur atom and yields the Bechgaard salts series (TMTSF) $)_{2} \mathrm{X}[87,101]$. The selenium series was at the start considered more promising compared to previous organic compounds, mainly because the metal insulator transition at ambient pressure only occurs below $20 \mathrm{~K}$ after high metallic conductivity have been attained [87].

In the two compounds of the series (TMTSF) ${ }_{2} \mathrm{PF}_{6}$ and (TMTSF) ${ }_{2} \mathrm{AsF}_{6}$, the transition occurs at $T_{c} \approx 12 \mathrm{~K}$ at 1 bar $[87,101,102]$. The transition early showed all the characteristics of SDW long-range ordering $[103,104]$. Similar SDW is also found in (TMTTF $)_{2} \mathrm{X}$ but at much higher values on the pressure scale. In (TMTTF $)_{2} \mathrm{PF}_{6}$ for example, about $40 \mathrm{kbar}$ of pressure is needed to reach a $T_{c} \sim 10 \mathrm{~K}$ (Fig. 1.2) $[12,105]$, whereas for $(\mathrm{TMTTF})_{2} \mathrm{Br}$ $[82,106]$ and $(\mathrm{TMTTF})_{2} \mathrm{BF}_{4}[107]$ about $10 \mathrm{kbar}$ and $27 \mathrm{kbar}$ must be applied, respectively.

The gradual emergence of a plasma edge in the $b$ direction below $100 \mathrm{~K}$ $[108,109]$, and the recovery of transverse metallic and longitudinal Fermi liquid $\left(\sim T^{2}\right)$ resistivity below $T^{\star}$ for (TMTSF $)_{2} \mathrm{PF}_{6}$ indicate that the transverse electron band motion has developed some coherence at the onset of the SDW transition. The mechanism of the instability will then naturally follow from the property of nesting of the Fermi surface based on the property $E_{-p}^{*}(\mathbf{k})=-E_{p}^{*}\left(\mathbf{k}+\mathbf{q}_{0}\right)$ of the spectrum (1.16) for a special wave vector $\mathbf{q}_{0}$, called the nesting vector. The relevance of the Fermi surface for the transition has been confirmed by the determination of the modulation wave vector of SDW by NMR [110], which coincides with the best nesting vector obtained by band calculations [8]. In the simplified model spectrum Eq. (1.16) for an approximate orthorhombic lattice, perfect nesting is found at $\mathbf{q}_{0}=\left(2 k_{F}, \pi, \pi\right)$. Deviations with respect to this ideal situation, however, are likely to be found in practice. This amounts to use the spectra

$$
E_{p}^{*}(\mathbf{k})=v\left(p k-k_{F}\right)-2 t_{\perp b}^{*} \cos k_{\perp b}-2 t_{\perp c}^{*} \cos k_{\perp c}-2 t_{\perp b}^{\prime} \cos 2 k_{\perp b},
$$

that contains small next-to-nearest-neighbor hopping $t_{\perp b}^{\prime}$ along the $b$ direction $\left(t_{\perp b}^{\prime} \ll t_{\perp b}\right)$. This leads to the modified nesting condition

$$
E_{p}^{*}(\mathbf{k})=-E_{-p}^{*}\left(\mathbf{k}+\mathbf{q}_{0}\right)+4 t_{\perp b}^{\prime} \cos 2 k_{\perp b},
$$

with $k_{\perp b}$ dependent nesting frustration.

The determination of the temperature scale for the SDW instability follows the analysis given in $\S 1.2 .2$, where the ladder result Eq. (1.24) becomes 


$$
\tilde{J}(T)=\frac{\tilde{J}^{*}}{1-\frac{1}{2} J^{*} \chi^{0}\left(\mathbf{q}_{0}, T\right)},
$$

where

$$
\pi v_{F} \chi^{0}\left(\mathbf{q}_{0}, T\right)=\ln \frac{T_{x^{1}}}{T}+\psi\left(\frac{1}{2}\right)-\Re \mathrm{e}\left\langle\psi\left(\frac{1}{2}+i \frac{t_{\perp b}^{\prime} \cos 2 k_{\perp b}}{\pi T}\right)\right\rangle_{k_{\perp b}},
$$

is the bare susceptibility in the presence of nesting frustration $t_{\perp b}^{\prime}(\psi(x)$ is the Digamma function and $\langle\ldots\rangle_{k_{\perp b}}$ is an average over $k_{\perp b}$ ) [111]. As mentioned previously, the above expressions differ slightly from previous meanfield approaches $[34,35,111,112]$, in that the contribution of intermediate electron-hole excitations to $\chi^{0}$ has been here restricted to an energy shell $\pm T_{x^{1}}$ (instead of $\pm E_{F}$ ) around the Fermi level. More energetic excitations extending up to the Fermi energy are one-dimensional in character and are governed by Eqs. (1.2) [17, 28,30,113]. The above Stoner form also neglects the finite coupling between electron-hole and electron-electron pairings, an interference that persists even below $T_{x^{1}}$. This approximate weak coupling description of SDW remains qualitatively correct, however, as long as $t_{\perp b}^{\prime}$ does not reach too large values, that is where the interference between densitywave and superconductivity can change the nature of the ground state (see $\S 1.4 .6)$.

The Stoner form (1.41) develops a singularity at the critical temperature $T_{c}$ that depends on $t_{\perp b}^{\prime}$, the main parameter that is standardly used to mimic the actual effect of pressure on the SDW state [34,35,111,114]. A finite $t_{\perp b}^{\prime}$ will then reduce $T_{c}$ with respect to the BCS limiting value $T_{c}^{0}=T_{x^{1}} e^{-2 / \tilde{J}^{*}}$ at perfect nesting (Eq. (1.25)), a feature of the model that was soon linked with experiments done under pressure $[101,102,115,116]$ (inset of Fig. 1.24). Besides the monotonic increase of $T_{x^{1}}$ and the decrease of $\tilde{J}^{*}$ under pressure, the detrimental influence of $t_{\perp b}^{\prime}$ on $T_{c}$ remains the most dominant effect.

The expression (1.41) predicts that at the approach of the critical value $t_{\perp b}^{\prime \text { cr }} \sim 0.7 T_{c}^{0}, T_{c}$ rapidly goes down to zero - though the possibility of SDW at incommensurate $\mathbf{q}_{0}$ and very low temperature may introduce a change in the slope of $T_{c}$ near $P_{c}$ [117]. To $t_{\perp b}^{\prime c r}$ will then correspond a critical pressure $P_{c}$ for the suppression of SDW; this variation of $T_{c}$ agrees with the characteristic pressure profile generally observed for (TMTSF $)_{2} \mathrm{X}$ at moderate pressure (Fig. 1.24) and for (TMTTF) $)_{2} \mathrm{X}$ at higher pressure (Figs. 1.2 and 1.3). The fitness of the model to describe the $T_{c}$ of the SDW state in (TMTSF) ${ }_{2} \mathrm{X}$ can be further assessed if one considers the influence of a transverse magnetic field on $T_{c}$. A perpendicular magnetic field $H \| \mathrm{c}^{*}$ tends to confine the motion of electrons along the chain direction and gradually restores better nesting conditions. This in turn increases $T_{c}$ with $H$ [111], consistently with early field dependent measurements of $T_{c}$ in (TMTSF) ${ }_{2} \mathrm{PF}_{6}$ [118]. 


\section{Intrusion of charge-density-wave order}

The reexamination of diffuse scattering X-ray patterns of (TMTSF) ${ }_{2} \mathrm{PF}_{6}$ by Pouget and Ravy [81,119] revealed the emergence, besides SDW, of a chargedensity-wave superstructure (CDW) at $T_{c}$; both having the same modulation vector $\mathbf{q}_{0}$. These results were subsequently confirmed by Kagoshima et al. [120], who also found a similar superstructure in (TMTSF) ${ }_{2} \mathrm{AsF}_{6}$, but with a weaker amplitude. These results came as a surprise since at variance with ordinary Peierls phenomena, the CDW order is not preceded by any lattice softening in the normal state (the $2 k_{F}$ diffuse scattering lines do exist at high temperature but their amplitudes become vanishingly small in the vicinity of $T_{c}$ in the normal state [54,119]). The CDW superstructure would then be entirely electronic in character with no lattice displacement involved.

In connection with these X-ray results, it is worth mentioning the earlier optical conductivity measurements of $\mathrm{Ng}$ et al. [121], on the isostructural member of the series (TMTSF) ${ }_{2} \mathrm{SbF}_{6}$. The results show the growth in the infrared of new phonon lines at $T_{c}$, precisely those usually expected for the excitations of a CDW superstructure; their temperature dependent intensity follows roughly the one of the SDW order parameter below $T_{c}$. CDW phonon lines in the far infrared conductivity have also been found in the metallic phase of (TMTSF) ${ }_{2} \mathrm{ClO}_{4}$ at low temperature [122], indicating that $2 k_{F}$-CDW and SDW correlations apparently coexist in the normal phase $[27,42]$.

On theoretical grounds the possibility for SDW and CDW to coexist has been analyzed recently by considering the redistribution of charge and spin in the unit cell, a possibility that emerges when its internal - two-molecules - structure and the long-range Coulomb interaction are taken into account. In the framework of extended Hubbard model, numerical and mean-field approaches show that charge and spin can be so rearranged in the unit cell that SDW, BOW and CDW can coexist $[59,79,123,124]$. Moreover, it was shown recently that when interchain Coulomb interaction is included, this can favor

- even for small amplitude - BOW and CDW correlations while not affecting SDW [125].

\subsubsection{Some features of the superconducting state}

\section{The superconducting transition}

For all cases of superconductivity in the $(\mathrm{TM})_{2} \mathrm{X}$ series, the first evidence has been provided by a drop of the resistivity below the critical temperature and the suppression of this drop under magnetic field. We shall focus the presentation on the two members of the (TMTSF) ${ }_{2} \mathrm{X}$ series, which have attracted most attention:

- i) (TMTSF $)_{2} \mathrm{PF}_{6}$, because this has been the first organic superconductor to be found by transport measurements [101], and subsequently confirmed by magnetic shielding [126,127], and also because the electronic properties 
of the 1-D electron gas on the organic stacks are only weakly (if at all) affected by the centrosymmetrical anions $\mathrm{PF}_{6}$. The finding of a very small and still non-saturating resistivity under ambient pressure reaching the value of $10^{-5} \Omega^{-1} \mathrm{~cm}^{-1}$ at $12 \mathrm{~K}$ triggered further pressure studies at a pressure of $9 \mathrm{kbar}$ in a dilution refrigerator, which led to the discovery of a zero resistance state below $1 \mathrm{~K}$ (see Fig. 1.19). The non saturation of the resistivity had been taken as a signature of superconducting precursor effects. We shall come again to this important question later.

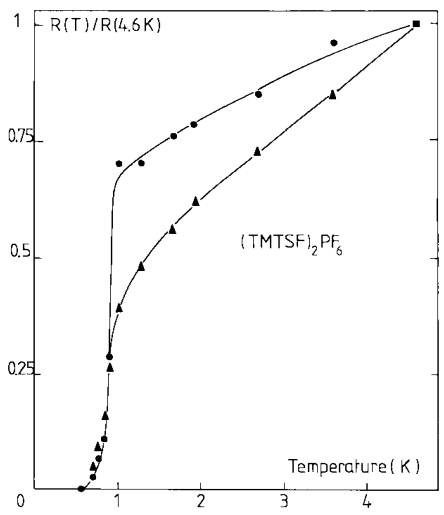

Fig. 1.19. (TMTSF $)_{2} \mathrm{PF}_{6}$, first observation of organic superconductivity under pressure. After Ref. [101]

- ii) (TMTSF) ${ }_{2} \mathrm{ClO}_{4}$, because it is the only member of the (TM) $)_{2} \mathrm{X}$ series displaying superconductivity at ambient pressure. However, the study of the superconducting state in (TMTSF) ${ }_{2} \mathrm{ClO}_{4}$ is meeting the problem of the $\mathrm{ClO}_{4}$ anions ordering at $24 \mathrm{~K}$, doubling the periodicity along the $b$ axis [60]. Consequently, great care must be taken to cool the sample slowly enough in order to reach a well anion-ordered state (R-state) at low temperature; otherwise superconductivity is faced to its great sensitivity to disorder, a very important feature for organic superconductors that will be discussed more extensively below.

Since (TMTSF $)_{2} \mathrm{ClO}_{4}$ is an ambient pressure superconductor the thermodynamic evidence of the phase transition has been obtained from specific heat on single crystals [128]. The electronic contribution to the specific heat of (TMTSF) ${ }_{2} \mathrm{ClO}_{4}$ in a $C_{e} / T$ vs $T$ plot (Fig. 1.21), dipslays a very large anomaly around $1.2 \mathrm{~K}[128]$. Above $1.22 \mathrm{~K}$, the total specific heat obeys the classical relation in metals $C / T=\gamma+\beta T^{2}$, where the Sommerfeld constant for electrons $\gamma=10.5 \mathrm{~mJ} \mathrm{~mol}^{-1} \mathrm{~K}^{-2}$, corresponding to a density of states at the Fermi level $N\left(E_{F}\right)=2.1$ states $\mathrm{eV}^{-1} \mathrm{~mol}^{-1}$ for the two spin directions [128]. 


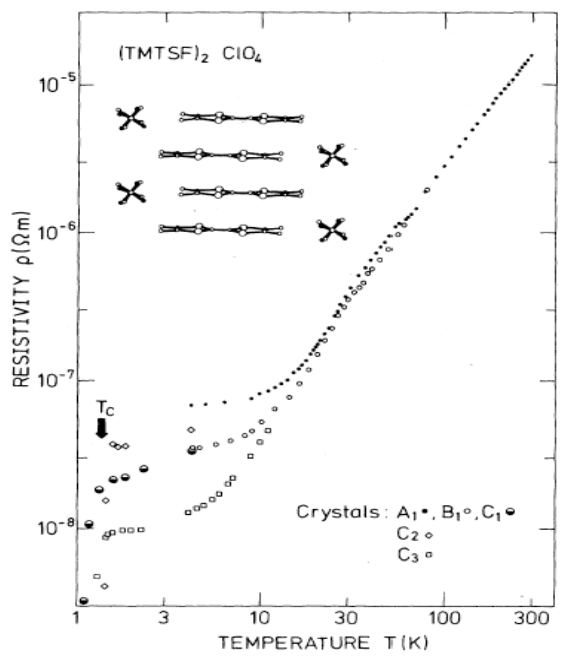

Fig. 1.20. (TMTSF $)_{2} \mathrm{ClO}_{4}$, first observation of organic superconductivity at ambient pressure. After Ref. [94]

The specific heat jump at the transition amounts then to $\Delta C_{e} / \gamma T_{c}=1.67$, i.e. only slightly larger than the BCS ratio for a $s$-wave superconductor. The behavior of $C_{e}(T)$ in the superconducting state leads to the determination of the thermodynamical critical field $H_{c}=44 \pm 2$ Oe and the quasi-particle gap $2 \Delta=4 \mathrm{~K} . T_{c}$ is depressed at a rate of $1.1 \mathrm{mK} / \mathrm{Oe}^{-1}$, when a magnetic field is applied along the $c^{*}$ axis [129]. Comparing the value of the density of states derived from the specific heat and the value of the Pauli susceptibility [43], lends support to a weak coupling Fermi liquid picture (at least in the low temperature range) [89].

Another confirmation of organic superconductivity has been provided by the measurement of thermal conduction in (TMTSF $)_{2} \mathrm{ClO}_{4}$ [130]. From the difference between thermal conductivity in magnetic field (larger than the critical field) and in zero applied field, the authors of Ref. [130] have been able to extract the electronic contribution of the thermal conductivity below $T_{c}$ down to about $T_{c} / 5$, Fig. (1.22). These data lead to a ratio $\Delta(0) / k_{B} T_{c}=2$ within the Bardeen Rickaysen and Teword (BRT) theory of the thermal conduction in the superconducting state [131]. Such a ratio is in fair agreement with the specific heat jump data mentioned above. The saturation of the electronic thermal conduction observed at low temperature on Fig.(1.22) is in favor of a well defined gap in the quasi-particle spectrum (possibly due to the interplay with the anion gap of this particular compound) but does not necessarily implies $s$-pairing for the orbital symmetry of the superconducting wave function (see the discussion in $§ 1.4 .6$ ). The results of thermal conductivity contrast with those NMR spin-lattice relaxation rate measure- 


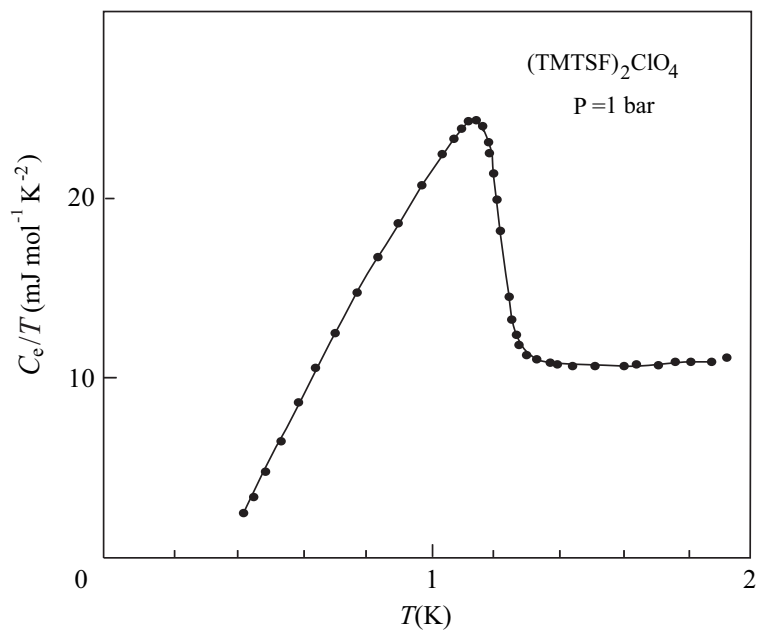

Fig. 1.21. Electronic contribution to the specific heat of (TMTSF $)_{2} \mathrm{ClO}_{4}$, plotted as $C_{e} / T$ versus $T$. After [128]

ments obtained earlier by Takigawa et al., on (TMTSF) ${ }_{2} \mathrm{ClO}_{4}$ [132], which show the absence of an Hebel Slichter peak at $T_{c}$ and a power law dependence $T_{1}^{-1} \propto T^{3}$ for protons NMR $\left({ }^{1} \mathrm{H}\right)$ - these two features being compatible with the existence of nodes for the superconducting gap [133]. A similar algebraic dependence on temperature for $T_{1}^{-1}$ has been found for ${ }^{77}$ Se nuclei in (TMTSF $)_{2} \mathrm{PF}_{6}$ above the critical pressure $P_{c}[134]$.

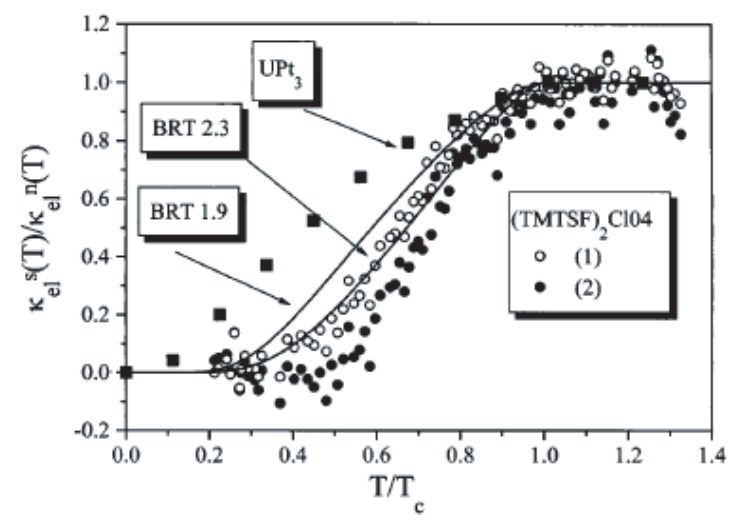

Fig. 1.22. (TMTSF $)_{2} \mathrm{ClO}_{4}$, normalized electronic thermal conductivity compared to the BRT theory and the data in the unconventional heavy fermion superconductor. After Ref. [130] 
The onset of superconducting order has also been detected by a shift of the muon precession frequency entering the superconducting state below $T_{c}$ under a field of $180 \mathrm{G}$ [135]. However, the supeconducting state is not accompanied by any enhancement of the muon relaxation rate according to the data of reference [135], and the recent zero field data of [136]. Such a behavior is at variance with the behaviour of $\mathrm{Sr}_{2} \mathrm{Ru}_{4}$ [137], in which the increase of the relaxation rate in a zero applied field suggests the development of spontaneous magnetic fields and is taken as a possible (but not unique) evidence for time-reversal symmetry breaking and triplet superconductivity in this oxide material.

\section{Critical fields}

The anisotropic character of the electronic structure already known from the anisotropy of the optical data in the normal phase, is reflected in a severe anisotropy of the critical fields $H_{c 2}$ measured along the three principal directions in (TMTSF) ${ }_{2} \mathrm{ClO}_{4}$ [138-141]. Early data in (TMTSF) ${ }_{2} \mathrm{ClO}_{4}$ [141] are not in contradiction with the picture of singlet pairing but no data were given below $0.5 \mathrm{~K}$, the temperature domain where it would be most rewarding to see how $H_{c 2}$ compares with the Pauli limit, when $H$ is perfectly aligned along

the $a$ and $b^{\prime}$ axes. This study has been revisited quite recently in perfectly aligned magnetic fields down to $0.2 \mathrm{~K}$ [142].

The linearity of the critical fields with temperature in the vicinity of the $T_{c}$ suggests an orbital limitation in the Ginzburg-Landau formalism for the critical field and rules out a Pauli limitation, which would favour a (1 $\left.T / T_{c}\right)^{1 / 2}$ dependence $[139,143]$.

Furthermore, in support to an early suggestion [143], the band structure parameters of (TMTSF) ${ }_{2} \mathrm{ClO}_{4}$ can explain the values and the anisotropies of the critical fields assuming the existence of nodes of the superconducting order parameter [142]. These results imply that critical fields values calculated without any contribution from the spin-orbit coupling can overcome the Pauli limit at low temperature by factors of two or more [144,145]. The situation for (TMTSF $)_{2} \mathrm{PF}_{6}$ may, however, be quite different as the possibility of a non homogenous superconducting phase in the vicinity of the critical pressure opens an other possibility for an enhancement of the superconducting critical fields, vide infra.

\section{Superconductivity and Pressure}

The pressure dependence of $T_{c}$ is admittedly a remarkable feature for the (TMTSF $)_{2} \mathrm{X}$ compounds since it is the pressure parameter that enabled organic superconductivity to be discovered. However, pressure suppresses also the superconducting phase very quickly. As far as (TMTSF) $)_{2} \mathrm{PF}_{6}$ is concerned, in the vicinity of the critical pressure the pressure coefficient amounts 


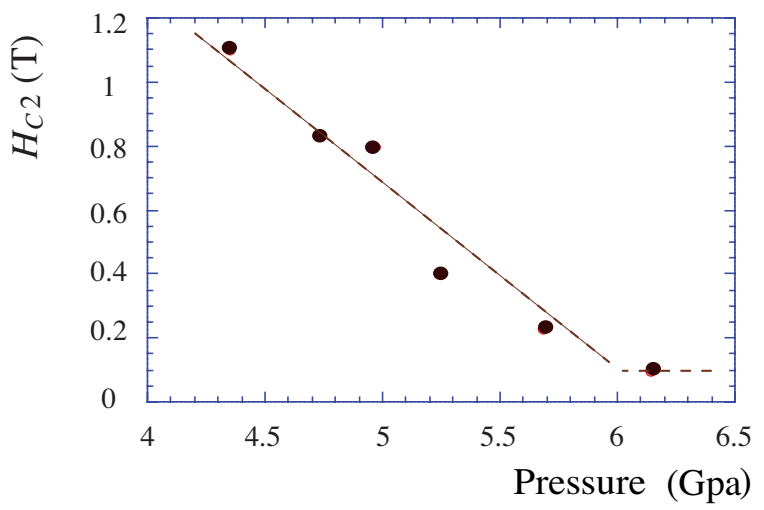

Fig. 1.23. (TMTTF $)_{2} \mathrm{PF}_{6}$, critical fields along $c^{\star}$ under pressure in the SDW/SC coexistence regime. The lines are guides to the eyes. After Ref. [146]

to $\delta \ln T_{c} / \delta P=11 \% \mathrm{kbar}^{-1}$ leading in turn to a Gruneisen constant for superconductivity $\delta \ln T_{c} / \delta \ln V=18$ at 9 kbar [147], with the compressibility data measured under pressure (at $16 \operatorname{kbar} \delta \ln V / \delta P=0.7 \% \mathrm{kbar}^{-1}$ ) [39] in the same compound. This value is indeed sizably larger than 7 , the value which is obtained in tin, the elemental superconductor exhibiting the strongest sensitivity to pressure [148]. A look at the (TMTSF) ${ }_{2} \mathrm{PF}_{6}$ phase diagram shows that the strong pressure dependence of $T_{c}$ is however restricted to the close vicinity of the border with the SDW phase.

The pressure coefficient of superconductivity in (TMTSF) ${ }_{2} \mathrm{ClO}_{4}$ is even more dramatic since then $\delta \ln T_{c} / \delta \ln V=36$ [149] using the compressibility of $1 \% \mathrm{kbar}^{-1}$ (this is the value measured for (TMTSF) ${ }_{2} \mathrm{PF}_{6}$ at ambient pressure [39], since to the best of our knowledge compressibility data for (TMTSF) ${ }_{2} \mathrm{ClO}_{4}$ are still missing). However, this remarkable sensitivity of $T_{c}$ in (TMTSF) ${ }_{2} \mathrm{ClO}_{4}$ might actually be related to the very specific problem of anion ordering in this compound as it has been suggested from the recent study on the sensitivity of $T_{c}$ against the presence of non-magnetic disorder [97]. Anion ordering reveals an uprise of the ordering temperature under pressure [149-151], which can be derived from the pressure dependence of a small kink in the temperature dependence of the resistivity, the signature of the ordering, moving from 24 up to $26.5 \mathrm{~K}$ under $1.5 \mathrm{kbar}$ and corroborated by studies at even higher pressures [152]. Together with this uprise, there exists a slowing down in the dynamics of the anions needed for the ordering. Hence, high pressure studies require a special attention to the cooling rate which must be kept low enough to allow anion ordering at low temperature. This may be the explanation for the discrepancy between high pressure data showing the signature of anion ordering up to $8 \mathrm{kbar}$ [151], and 
the absence of ordering claimed from the interpretation of magneto-angular oscillations [153].

\section{SDW-SC coexistence}

A situation of non-homogenous superconductivity have been clearly identified near the border between SDW and Superconductivity in the (TMTSF $)_{2} \mathrm{X}$ phase diagram [116]. At high pressure $(P>9.4 \mathrm{kbar})$, the superconducting phase emerges from a metallic state and can be reasonably thought of as homogeneous with a critical current density along the $a$-axis $J_{c}=$ $200 \mathrm{~A} \mathrm{~cm}^{-2}$. Below this critical pressure, there exists a pressure domain for $(\mathrm{TMTSF})_{2} \mathrm{PF}_{6}(\approx 1 \mathrm{kbar}$ wide) in which a superconducting signature is observed at a nearly pressure independent temperature below the onset of a SDW instability, where the critical current density is greatly reduced, $J_{c} \approx 10 \mathrm{~A} \mathrm{~cm}^{-2}$. This feature points in favor of a coexistence of SDW and SC macroscopic domains consisting of metallic (SC) tubes parallel to the $a$ axis (Fig. 1.24). The existence of coexisting macroscopic regions of SDW

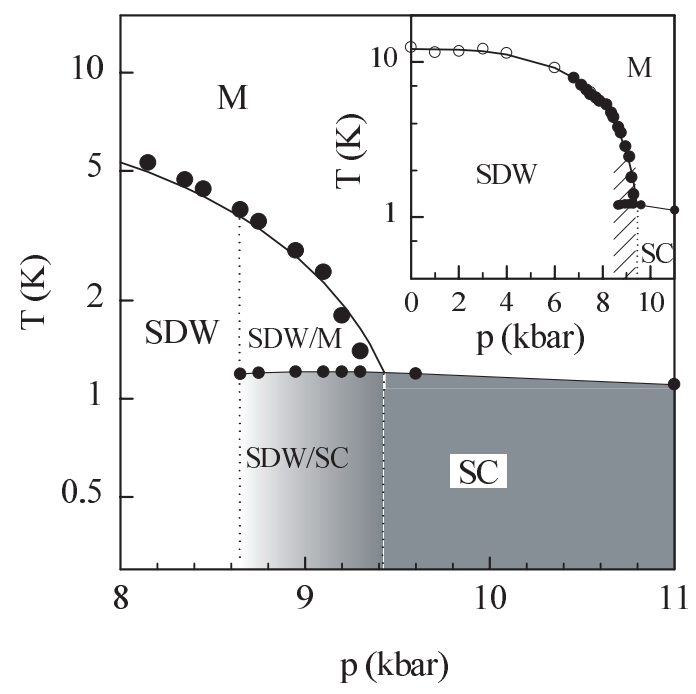

Fig. 1.24. Coexistence between SDW and Superconductivity in (TMTTF $)_{2} \mathrm{PF}_{6}$ in the vicinity of the criticial pressure for suppression of the SDW ground state. After Ref. [116]

and SC order is also supported by a recent NMR investigation performed at a pressure slightly lower than the critical pressure for the establishment of the homogenous state [154]. This latter study has enabled a quantification of the relative volume fractions in the SDW-metallic regime using the proton NMR linewidth as the local probe. A related consequence of the existence 
of macroscopic insulating domains in the superconducting phase allowing a channeling of the lines of force in the material is the large increase of the upper critical field $H_{c 2}$ [134], which had already been reported long time ago in (TMTSF $)_{2} \mathrm{PF}_{6}$ with the second confirmation of organic superconductivity [155], and also later in (TMTSF) ${ }_{2} \mathrm{AsF}_{6}[102]$.

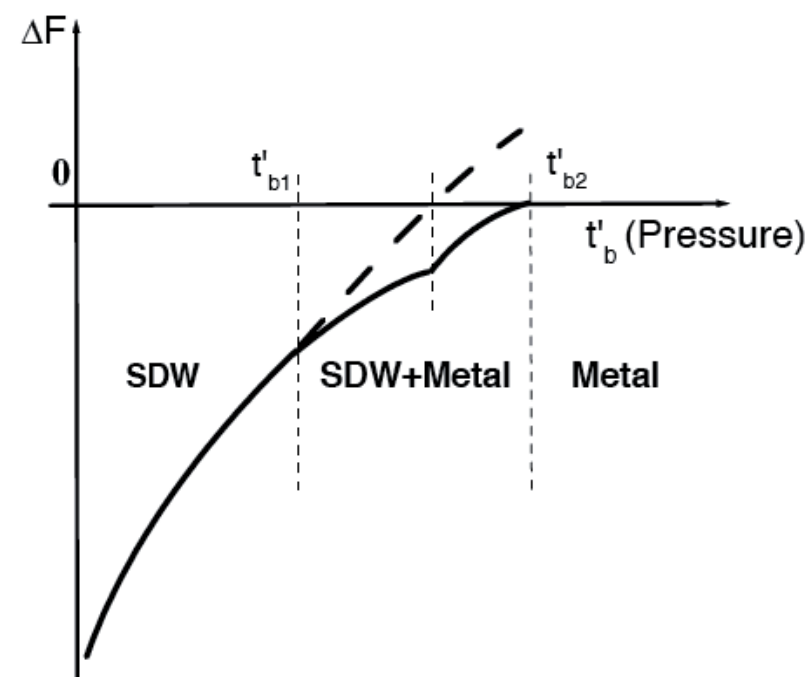

Fig. 1.25. Sketch of the theory showing from the free energy vs the parameter $t_{b}^{\prime}$, the region of coexistence between SDW and Superconductivity in the vicinity of the criticial pressure [116]. $t_{b}^{\prime}\left(\equiv t_{\perp b}^{\prime}\right.$ in the text) parametrizes the deviation from the perfect nesting of the Q-1-D Fermi surface. After Ref. [116]

It must be kept in mind that the slab formation in the vicinity of the SDW state with the formation of insulating domains is not related to the penetration of the magnetic field in a type II superconductor but the result from a competition between insulating and conducting phases. The claim for the existence of tubular domains $\| a$ is based on an analysis of the resistance in the vicinity of the critical pressure at low temperature above $T_{c}$, and of the critical currents in the superconducting state [116]. The theoretical approach relies on a variational model leading to an inhomogenous phase with an energy lower than the energy of the homogenous states (Metallic or Insulating SDW). Since it is the transverse $b$ parameter that is expected to govern the respective stability of the SDW and metallic phases at a pressure lower than the critical one but close enough to the homogenous critical line the formation of macroscopic metallic domains with a smaller $b$ is energetically favored in-between $b$ expanded SDW domains [156]. Similarly, at pressures larger than the critical pressure, insulating SDW domains should be present between metallic regions (see Fig. 1.25). This interpretation is at variance 
with a model based on similar experimental data for the critical fields in the coexistence regime in which the formation of thin superconducting slabs perpendicular to $a$ sandwiched between SDW insulating domains is the result of a self organization process taking advantage of the largest field penetration length perpendicular to the direction of the field [134].

An other approach has been taken by Gorkov and Grigoriev for the interpretation of the SDW/SC coexistence regime in (TMTSF) $)_{2} \mathrm{PF}_{6}$ [157]. This model predicts the existence of soliton domain walls in the SDW phase close to the critical pressure between the uniformly gapped SDW phase and the SC phase. As these domain walls should be perpendicular to the molecular stacking axis, a strong anomaly of the transport anisotropy could be anticipated related to the formation of conducting slabs perpendicular to the $a$ axis in the coexistence pressure regime. However, no such anomaly has been detected experimentally [158].

Recent data obtained with (TMTSF $)_{2} \mathrm{PF}_{6}$ under very high pressure, where the coexistence regime is much broader than for (TMTSF) $)_{2} \mathrm{PF}_{6}$, have clearly shown that the critical field for $H \| c^{*}$ is enhanced by a factor 10 at the border with the SDW phase. In this pressure domain, it can reach $1 \mathrm{~T}$, (Fig. 1.23), while it amounts to about $0.1 \mathrm{~T}$ at very high pressure when the superconducting state is homogenous [146], a value similar to the observation in the R-state of (TMTSF) ${ }_{2} \mathrm{ClO}_{4}$.

Finally, an experimental study performed in (TMTSF) ${ }_{2} \mathrm{ReO}_{4}$ under pressure has revealed the existence of conducting filaments parallel to $a$ in the pressure domain close to $10 \mathrm{kbar}$ when metallic and insulating domains coexist at low temperature as a consequence of two coexisting anion orders in this material [159].

\section{Superconductivity and non magnetic defects}

It is the remarkable sensitivity of organic superconductivity to irradiation $[160,161]$ that led Abrikosov to suggest the possibility of triplet pairing in these materials [162]. Although irradiation was recognized to be an excellent method for the introduction of defects in a controlled way [163], defects thus created can be magnetic [164], and the suppression of superconductivity by irradiation induced defects as a signature of non-conventional pairing must be taken with 'a grain of salt' since local magnetic moments can also act as strong pair-breakers on $s$-wave superconductors. Several routes have been followed to introduce an intrinsically non-magnetic perturbation modulating the potential seen by the carriers on the organic stacks. Non magnetic disorder has been achieved substituting TMTTF for TMTSF on the cationic stacks of $(\mathrm{TM})_{2} \mathrm{X}$ salts with $\mathrm{PF}_{6}[7]$ and $\mathrm{ClO}_{4}$ salts [165]. However, in both situations cationic alloying induces drastic modifications of the normal state electronic properties since the SDW transition of (TMTSF $)_{2} \mathrm{PF}_{6}$ is quickly broadened and pushed towards higher temperature upon alloying [166]. 
Leaving the cation stack uniform, scattering centers can also be created on the anion stacks with the solid solution (TMTSF) ${ }_{2} \mathrm{ClO}_{4(1-\mathrm{x})} \mathrm{ReO}_{4 \mathrm{x}}$, where Tomić et al., first mentioned the suppression of superconductivity upon alloying with a very small concentration of $\mathrm{ReO}_{4}$ anions [167,168]. In the case of a solid solution with tetrahedral anions such as $\mathrm{ClO}_{4}$ or $\mathrm{ReO}_{4}$, one is confronted to two potential sources of non-magnetic disorder that act additively on the elastic electronic lifetime according to the Mathiessen's law. First the modulation due to the different chemical natures of the anions and second a disorder due to a progressive loss of long-range ordering at $T_{\mathrm{AO}}$ in the $(\mathrm{TM})_{2} \mathrm{X}$ solid solution, although $\mathrm{X}$-ray investigations have revealed that long-range order is preserved up to $3 \% \mathrm{ReO}_{4}$ with a correlation length $\xi_{a}>$ $200 \AA$ [169]. Studies of superconductivity in (TMTSF) ${ }_{2} \mathrm{ClO}_{4}$ performed under extremely slow cooling conditions have shown that $T_{c}$ is a fast decreasing function of the non-magnetic disorder [97], where the residual resistivity along the $c^{*}$ axis has been used for the measure of the disorder in the alloys with different concentrations (Fig. 1.26). It must be emphasized that the residual resistivity is derived from a fit of the temperature dependence of the normal state resistivity according to a Fermi liquid model below the anion ordering temperature of $24 \mathrm{~K}$, namely $\rho(T)=\rho_{0}^{-}+A T^{2}$. This treatment of the resistivity below $10 \mathrm{~K}$ or so, allows us to remove the influence of superconducting precursor effects above the ordering temperature.

The suppression of $T_{c}$ is clearly related to the enhancement of the scattering rate in the solid solution. Since the additional scattering cannot be ascribed to magnetic scattering according to the EPR checks showing no additional traces of localized spins in the solid solution, the data in figure (1.26) cannot be reconciled with the picture of a superconducting gap keeping a constant sign over the whole $\left( \pm k_{F}\right)$ Fermi surface. They require a picture of pair breaking in a superconductor with an unconventional gap symmetry. The conventional pair breaking theory for magnetic impurities in usual superconductors has been generalized to the case of non-magnetic impurities in unconventional materials and the correction to $T_{c}$ obeys the following relation $[171,172]$,

$$
\ln \left(\frac{T_{c}^{0}}{T_{c}}\right)=\psi\left(\frac{1}{2}+\frac{\alpha T_{c}^{0}}{2 \pi T_{c}}\right)-\psi\left(\frac{1}{2}\right),
$$

with $\psi(x)$ being the Digamma function, $\alpha=\hbar / 2 \tau k_{B} T_{c}^{0}$ the depairing parameter, $\tau$ the elastic scattering time and $T_{c}^{0}$ the limit of $T_{c}$ in the absence of any scattering. From the data in figure (1.26), the best fit leads to $T_{c}^{0}=1.57 \mathrm{~K}$ and a critical scattering for the suppression of superconductivity of $1 / \tau_{\mathrm{cr}}=1.85 \mathrm{~cm}^{-1}$. Accordingly, $1 / \tau$ amounts to $0.56 \mathrm{~cm}^{-1}$ in the pristine (TMTSF) ${ }_{2} \mathrm{ClO}_{4}$ sample. Such a value for the inverse carrier life time is admittedly significantly smaller than the predicted width at half height namely, $1 / \tau \approx 2 \mathrm{~cm}^{-1}$ assuming a classical Drude behaviour involving the temperature dependence of the DC conductivity and the longitudinal plasma 


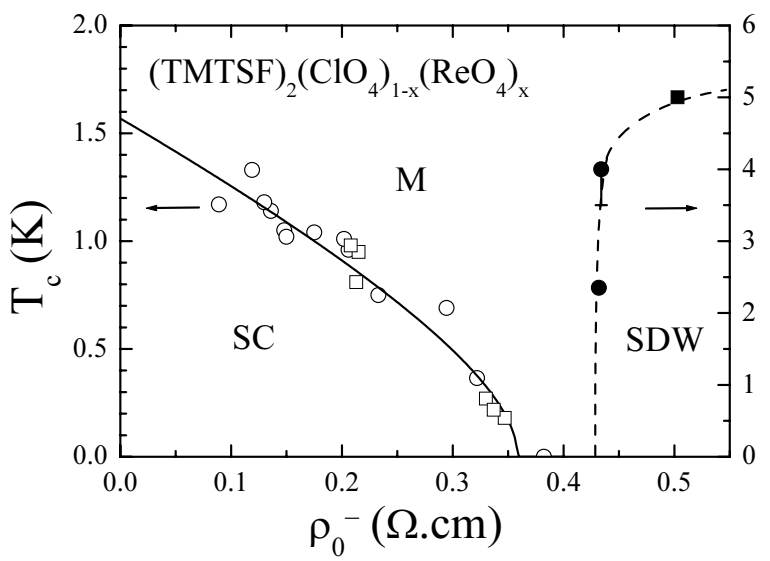

Fig. 1.26. Phase diagram of $(\mathrm{TM})_{2} \mathrm{X}$ governed by non magnetic disorder. All open circles refer to the very slowly cooled samples in the R-state with different $\mathrm{ReO}_{4}^{-}$contents. Open squares are data from the same samples corresponding to slightly larger cooling rates although keeping a metallic behavior above $T_{c}$. A $10 \%$ sample with $\rho_{0}^{-} \approx 0.32 \Omega$.cm has provided four different $T_{c}$ depending on the cooling rate. One sample $(8 \%)$ did not reveal any ordering down to $0.1 \mathrm{~K}$. These data show that the residual resistivity is a better characterization for the disorder than the nominal $\mathrm{ReO}_{4}^{-}$concentration. Full dots (15 and 17\%) are relaxed samples exhibiting a SDW ground state. The vertical bar is the error bar for a sample in which a maximum of the logarithmic derivative could not be clearly identified and therefore the actual SDW temperature should lie below $4 \mathrm{~K}$, the temperature of minimum resistivity. The full square is the Q-state of a $6 \%$ sample. The continuous line at the Metal-SC transition is the best fit of the data with the diGamma function model providing $T_{c}^{0}=1.57 \mathrm{~K}$. The dashed line at the Metal-SDW transition is only a guide for the eye. After Ref. [170]

frequency [49]. The present derivation of the electron life time compares fairly well with far infrared optical measurements leading to a zero frequency conductivity peak with a width less than $2-4 \mathrm{~cm}^{-1}[92,95]$. Our results support the existence of a very narrow zero frequency peak carrying a minor fraction of the total spectral weight, which is probably the signature of a correlated low dimensional Fermionic gas.

The sensitivity of $T_{c}$ to non-magnetic disorder cannot be reconciled with a model of conventional superconductors. The gap must show regions of positive and negative signs on the Fermi surface, which can be averaged out by a finite electron lifetime due to elastic scattering. As these defects are local, the scattering momentum of order $2 k_{F}$ can mix $+k_{F}$ and $-k_{F}$ states and therefore the sensitivity to non-magnetic scattering is still unable to tell the difference between $p_{x}$ and $d$ orbital symmetries for the superconducting wave function. A noticeable progress could be achieved paying attention to the spin part of the wave function. In the close vicinity of $T_{c}$, orbital limi- 
tation for the critical field is expected to prevail and therefore the analysis of the critical fields close to $T_{c}$ does not imply a triplet pairing [143]. When the magnetic field is oriented along the intermediate $b$-axis violations of the Pauli limitation have been claimed in (TMTSF) ${ }_{2} \mathrm{PF}_{6}[144]$ and recently in (TMTSF $)_{2} \mathrm{ClO}_{4}$ superconductors [145]. However, it must be kept in mind that in all these experiments under transverse magnetic field along the $b^{\prime}$ axis, the electronic structure is profoundly affected by the application of the field which tends to localize the electrons as shown by the normal state crossing over from a metallic to an insulating state when investigated with a current along $c^{*}[142]$. Furthermore it is still unclear whether the superconducting phase remains uniform under very strong transverse field $[173,174]$.

The nature of the superconducting coupling in $(\mathrm{TM})_{2} \mathrm{X}$ conductors is still intensively studied and debated. The absence of temperature dependence of the ${ }^{13} \mathrm{C}$ Knight shift through the critical temperature at a pressure where (TMTSF $)_{2} \mathrm{PF}_{6}$ is superconducting implies a triplet pairing [134]. However, the question of sample thermalization during the time of the NMR experiment has been questioned [175] and this result will have to be reconfirmed. The nature of the coupling in $(\mathrm{TM})_{2} \mathrm{X}$ superconductors has not yet reached a consensus. This is due in part to the lack of unambiguous experimental data for samples exhibiting superconductivity in the very low temperature region. This is at variance with the singlet coupling found in 2D organic superconductors with a $T_{c}$ in the $10 \mathrm{~K}$ range as clearly indicated by the Knight shifts measurements in the superconducting state $[176,177]$. It can be noticed that in spite of the established singlet coupling, the critical fields $H_{c 2}$ of $2 \mathrm{D}$ superconductors can also greatly exceed the paramagnetic limit in the parallel geometry [178-180].

\section{An approach to the mechanism of superconductivity}

Given the experimental uncertainty about the nature of superconductivity in the Bechgaard and Fabre salts, it is therefore still premature to privilege the triplet scenario for pairing over the singlet one. In any case, however, superconductivity certainly differs from what is commonly seen in ordinary metals and this raises the question of the possible causes of unconventional pairing in these materials [181]. This problem perplexed almost every one in the field from the start, since the requirements for a traditional phononmediated mechanism for pairing are apparently not met [182]. Antiferromagnetism that completely surrounds superconductivity in the phase diagram represents the main obstacle for an effective attraction mediated by phonons to take place. Superconductivity is indeed invariably replaced by an SDW instability whether one moves backward on the pressure scale or whether at fixed pressure $P>P_{c}$, one moves along the magnetic field axis $\mathbf{H}\left(\| c^{*}\right)$, where a cascade of field-induced SDW states is found [183]. (TMTSF) ${ }_{2} \mathrm{ClO}_{4}$ is another example that illustrates how close $(\mathrm{TM})_{2} \mathrm{X}$ are to the threshold of a SDW instability at $P>P_{c}$. This compound presents an anion ordering 


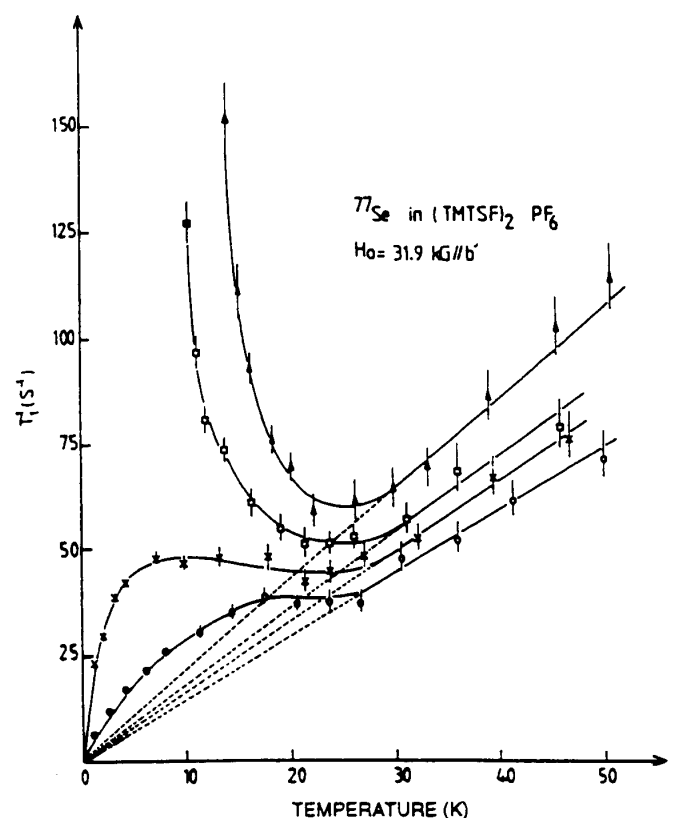

Fig. 1.27. Temperature dependence of the nuclear spin relaxation rate $T_{1}^{-1}$ of (TMTSF) ${ }_{2} \mathrm{PF}_{6}$ at $\mathrm{P}=1$ bar (triangles), 5.5kbar (squares), 8kbar (crosses) and 10 kbar (full circles). Deviations with respect to a linear temperature behavior result from antiferromagnetic fluctuations. After Ref. [52]

on slow cooling and is already a superconductor at ambient pressure $\left(P_{c}<1\right.$ bar, Fig. 1.20); it develops an SDW instability either beyond a critical alloying $[167,170]$ (Fig. 1.26), or by just cranking up the cooling rate [167,184,185]. Additional experimental weight supporting underlying coupling conditions for SDW comes from the properties of the normal state at $P>P_{c}$. NMR experiments show indeed a strong enhancement of the spin-lattice relaxation rate $T_{1}^{-1}$ as a function of temperature, revealing the existence of strong antiferromagnetic spin fluctuations in a very broad temperature domain of the metallic state above the superconducting $T_{c}$ (Fig. 1.27) $[27,42,52,186]$. Therefore all this goes to show that even in the presence of superconductivity, the interactions in $(\mathrm{TM})_{2} \mathrm{X}$ at $P>P_{c}$ remain repulsive and favorable to a SDW state, only nesting conditions are apparently changing in each case.

According to the model described in $\$ 1.4 .5$, these fluctuations are made at the microscopic level of electron-hole pairs at $\left(\mathbf{k}, \mathbf{k} \pm \mathbf{q}_{0}\right)$. These will then coexist with electron-electron (and hole-hole) pairing at $(\mathbf{k},-\mathbf{k})$, namely those responsible for the superconducting instability of the normal state at $P>P_{c}$. Since these two different pairings refer to the same electronic excitations around the Fermi surface, there will be some intrinsic dynamics or interfer- 
ence between them. We already came up against the problem of interfering pairing instabilities in the one-dimensional case $(\S 1.2)$. For repulsive interactions and perfect nesting at $2 k_{F}$, we have seen that interference between electron-hole and electron-electron pairing is maximum for a $1 \mathrm{D}$ - two points - Fermi surface and enters as a key ingredient in the formation of either a Luttinger liquid or a Mott insulating phase at commensurate filling [13-15]. At finite $t_{\perp b}$ and for temperature well below $T^{\star}$, however, the outcome differs and may provide a logical link between SDW and superconductivity.

The connection between superconductivity and density-wave correlations in isotropic systems goes back to the work of Kohn and Luttinger in the mid sixties [187]. They showed that the coupling between electron-hole (densitywave) and electron-electron correlations, albeit very small, is still present for a spherical Fermi surface. In this isotropic limit, $2 k_{F}$ Friedel (charge) fluctuations act as a oscillating pairing potential for electrons giving rise to a purely electronic mechanism for superconductivity at large angular momentum. Emery suggested that this non-phonon mechanism should be working in the spin sector as well, being boosted by the proximity of a SDW state in the quasi-1D geometry in order to yield experimentally reachable $T_{c}$ [188] an effect that was early on confirmed in the framework of renormalized meanfield theory $[31,189,190]$ and various RPA approaches [191-193]. However, these approaches amount to extract an effective superconducting coupling from short-range density-wave correlations, which in turn serves as the input of a ladder diagrammatic summation. It turns out that the ladder theory, as a single channel approximation, neglects the quantum interference between the different kinds of pairings, and as such it cannot capture the dynamical emergence of superconductivity.

Because of the finite value of $t_{\perp b}$, interference becomes non uniform along the Fermi surface. This introduces a momentum dependence in the scattering amplitudes, which can be parametrized by the set of transverse wave vectors for in going $\left(k_{\perp 1} k_{\perp 2}\right)$ and outgoing $\left(k_{\perp 1}^{\prime} k_{\perp 2}^{\prime}\right)$ electrons (here $\left.k_{\perp} \equiv k_{\perp b}\right)$. The generalization of the $1 \mathrm{D}$ scaling equations Eqs. (1.2) to now $k_{\perp}$-dependent interactions $g_{i=1,2,3}\left(k_{\perp 1}^{\prime} k_{\perp 2}^{\prime} k_{\perp 2} k_{\perp 1}\right)$ in the quasi-1D case, where both $t_{\perp b}$ and $t_{\perp b}^{\prime}$ are present, has been worked out recently $[114,125,194,195]$. The results can be put in the following schematic form:

$$
\begin{aligned}
\partial_{\ell} g_{i}\left(k_{\perp 1}^{\prime} k_{\perp 2}^{\prime} k_{\perp 2} k_{\perp 1}\right) & =\sum_{k_{\perp}} \sum_{n, n^{\prime}=1}^{3}\left\{\epsilon_{C, i}^{n n^{\prime}} g_{n}\left(\left\{k_{\perp}\right\}\right) g_{n^{\prime}}\left(\left\{k_{\perp}\right\}\right) \mathcal{L}_{C}^{\prime}\left(k_{\perp}, q_{C \perp}\right)\right. \\
& \left.+\epsilon_{P, i}^{n n^{\prime}} g_{n}\left(\left\{k_{\perp}\right\}\right) g_{n^{\prime}}\left(\left\{k_{\perp}\right\}\right) \mathcal{L}_{P}^{\prime}\left(k_{\perp}, q_{P \perp}, t_{\perp b}^{\prime}\right)\right\}, \quad(1.4
\end{aligned}
$$

Here $\mathcal{L}_{C, P}^{\prime}=\partial_{\ell} \mathcal{L}_{C, P}$ where $\mathcal{L}_{C, P}$ are the Cooper (electron-electron) and Peierls (electron-hole) loops, with $q_{C, P \perp}$ as their respective $\left\{k_{\perp}\right\}$-dependent transverse momentum variables, and $\epsilon_{C, P, i}^{n n^{\prime}}= \pm 1$, or 0 . By integrating these flow equations, the singularities shown by interactions signal instabilities of the normal state at a critical temperature $T_{c}$. The nature of ordering is deter- 


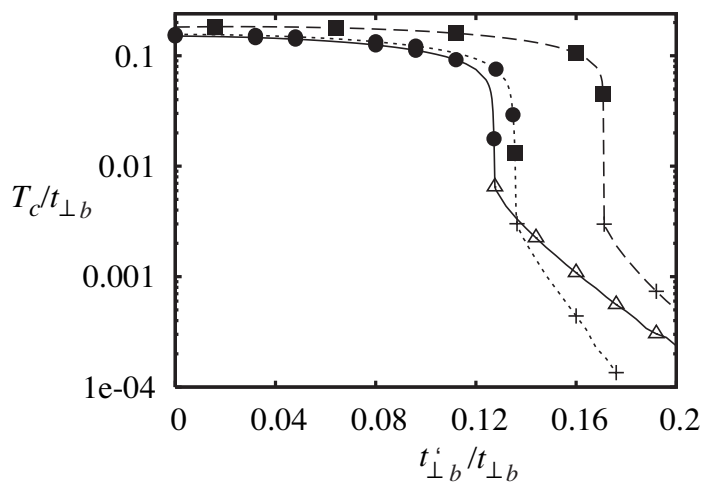

Fig. 1.28. Calculated phase transition temperature of the quasi-one-dimensional electron gas model as a function of the nesting deviation parameter $t_{\perp b}^{\prime}$ for repulsive intrachain interactions $g_{1,2,3}$. Continuous line $\left(\tilde{g}_{1}^{\perp}=0\right)$, dotted line $\left(\tilde{g}_{1}^{\perp}=0.11\right)$ and dashed line $\left(\tilde{g}_{1}^{\perp}=0.14\right.$.). After Nickel et al., Ref. [125]

mined by the profile of interactions in $\left\{k_{\perp}\right\}$ space, which in turn corresponds to a divergence of a given order parameter susceptibility $\chi_{\mu}$. Feeding these equations with a realistic set of bare parameters for the repulsive intrachain interactions $g_{i}$ and the band parameters $t_{\perp b}$ and $E_{F}$ in $(\mathrm{TM})_{2} \mathrm{X}$, it is possible to follow the instabilities of the normal state as a function of the nesting deviations parameter $t_{\perp b}^{\prime}$, which simulates the main influence of pressure in the model $[114,125,194-196]$.

Thus at perfect nesting, when $t_{\perp b}^{\prime}=0$, the normal state develops an SDW instability at $T_{c}^{0} \sim 20 \mathrm{~K}$, which for small Umklapp scattering corresponds to the range of $T_{c}$ expected in most of (TMTSF) $)_{2} \mathrm{X}$ at ambient pressure and (TMTTF $)_{2} \mathrm{X}$ at relatively high pressure. The range of $T_{c}$ roughly squares with the one obtained in the single channel approximation with no interference below $T_{x^{1}}$ (§1.4.5). As $t_{\perp b}^{\prime}$ increases, $T_{c}$ is gradually decreasing until the critical range $t_{\perp b}^{\prime \text { cr }} \simeq 0.8 T_{c}^{0}$ is reached where the SDW is suppressed (full circles, Fig. 1.28). The metallic phase remains unstable at finite temperature, however, but the instability now takes place in the superconducting channel (open triangles, Fig. 1.28). The order parameter is of the form $\Delta\left(k_{\perp}\right)=\Delta \cos k_{\perp}$ and has nodes at $k_{\perp} \pm \pi / 2$; it corresponds to a interstack singlet or $d_{x^{2}-y^{2} \text {-wave }}$ pairing. Therefore for repulsive intrachain interactions, an attraction between electrons can be dynamically generated from the interference between Cooper and Peierls scattering channels. The attraction between carriers on neighboring chains can be seen as being mediated by spin fluctuations. The fact that SC $d$ and SDW instability lines meet at the maximum of the superconducting $T_{c} \sim 1 \mathrm{~K}$ and that the ratio $T_{c}^{0}(\mathrm{SC} d) / T_{c}^{0}(\mathrm{SDW}) \sim 1 / 20$, together with their respective $t_{\perp b}^{\prime}$ dependence are worth noticing features in regard to the experimental phase diagram (Fig. 1.3). 
Regarding the possible symmetries of the superconducting order parameter, an analysis of the momentum dependence of the scattering amplitude $g_{i}\left(\left\{k_{\perp}\right\}\right)$ reveals that for the electron gas model defined with only intrachain repulsive interactions, the strongest superconducting instability is invariably found in the singlet SC $d$-wave channel $[114,196]$. Triplet superconductivity in the $p_{x}$ channel, which has been proposed on phenomenological grounds as a possible candidate to describe superconductivity in the Bechgaard salts [197], is strongly suppressed. In effect, the triplet $\mathrm{SC} p_{x}$ superconductivity, which has a gap order parameter $\Delta_{r}=r \Delta$ with $r=\operatorname{sign} k_{x}$, is an intrachain pairing that is subjected to the strongest repulsive part of the oscillating potential produced by SDW correlations [189]. More favorable conditions for triplet pairing do exist but they take place at higher angular momentum, in the interchain $f$-wave channel with a order parameter $\Delta_{r}\left(k_{\perp}\right)=r \Delta \cos k_{\perp}$, a possibility that was shown to come out from the mean-field analysis [198]. However, for intrachain repulsive couplings alone the renormalization group analysis show that the amplitude of triplet correlations are always subordinate to those of the $\mathrm{SC} d$-wave channel, which yields the highest superconducting $T_{c}[125,196]$.

Following the Kohn-Luttinger picture, triplet pairing at high angular momentum is actually connected to charge-density-wave fluctuations [187]. The presence of a CDW superstructure that coexists with a SDW state in the Bechgaard salts (see $\S 1.4 .5$ ) has in this respect stressed their importance in these salts close to $P_{c}$. Following the example of most quasi-onedimensional systems in which a CDW superstructure is found [199, 200], interchain Coulomb interaction is a physically relevant coupling that must be taken into account in the presence of charge correlations. By including, besides the $g_{i}$, interchain backward $\left(g_{1}^{\perp}\right)$, forward $\left(g_{2}^{\perp}\right)$ and Umklapp $\left(g_{3}^{\perp}\right)$ scattering amplitudes, one defines the so-called extended electron gas model $[201,202]$. For realistic repulsive couplings, this model allows us to expand the range of possibilities of both superconducting and density-wave long-range orders. The RG solution of Eqs. (1.44) in the $T-t_{\perp b}^{\prime}$ phase diagram shows that $g_{1}^{\perp}$ plays a key role on the one hand, in the stability of SDW and SC $d$ orders, and on the other hand in the emergence of triplet superconductivity and CDW order [125, 194]. The RG results depicted in Fig. 1.29 indeed show that beyond a relatively small threshold in $g_{1}^{\perp}, \mathrm{SC} d$ long-range order is no longer stable and the instability of the normal state is rather found in the interchain triplet $f$-wave channel above a critical $t_{\perp b}^{\prime}$. The SCf $T_{c}$ are comparable to $\mathrm{SC} d$ but show stronger 'pressure' coefficient along the $t_{\perp b}^{\prime}$ axis (Fig. 1.28). The results also show that the strength of SDW correlations remain essentially unaffected and can still dominate the normal phase in addition to CDW correlations, whose amplitude grows with the strength interchain coupling. Although SDW order can precede the triplet SCf instability along the $t_{\perp b}^{\prime}$ 'pressure' scale near the interchain coupling threshold, it is very close in stability with a CDW superstructure (Figs. 1.28 and 1.29). 


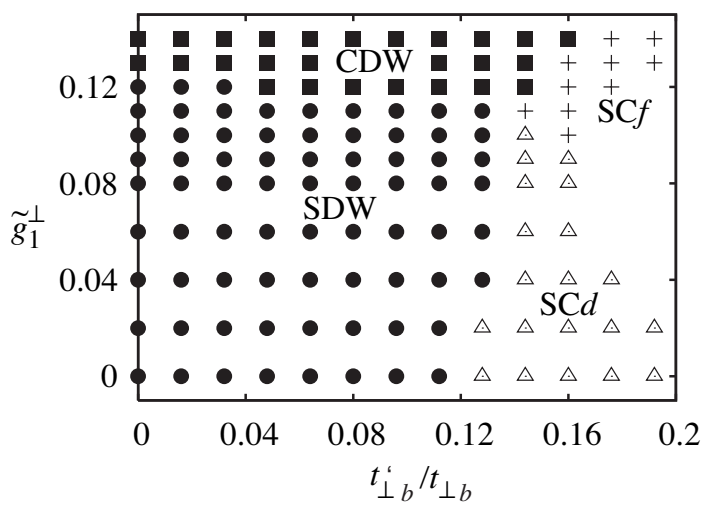

Fig. 1.29. Calculated phase diagram of the extended quasi-one-dimensional electron gas model for repulsive couplings. After Nickel et al., Ref. [125]

Given the observation of a close proximity between SDW and CDW in the phase diagram of the Bechgaard salts, it follows that not only singlet SCd but also $\mathrm{SC} f$ are serious candidates for the description of superconductivity in these materials. Since many parameters of the model are likely to change under pressure besides $t_{\perp b}$, this puts some haziness about how one actually moves in the phase diagram of Fig. 1.29, and in turn on the most stable type of superconductivity in these compounds.

\subsection{Conclusion and outlook}

In this brief account we went through the main physical properties of the Bechgaard and Fabre salts series of organic superconductors. The global phase diagram that was gradually built over the years around these two series of compounds under either hydrostatic or chemical pressure stands out as a model of unity for the physics of low dimensional correlated systems. Much effort went to explain the multifaceted phase diagram of $(\mathrm{TM})_{2} \mathrm{X}$ as a whole, an attempt that also proved to be an active quest of unity for the theory.

In this respect, while the theoretical description of the spin-Peierls and antiferromagnetic instabilities in the phase diagram of $(\mathrm{TM})_{2} \mathrm{X}$ does not meet any serious conceptual difficulty, their strong competition at the putative quantum critical point on the pressure scale is to our knowledge without precedent in the field of low dimensional compounds. Although the quantum criticality that is behind this competition would certainly gain to be further clarified on experimental ground, its comprehension clearly challenges the traditional framework of critical phenomena and fosters some new conceptual focus in unifying antiferromagnetism and a lattice distorted spin liquid phase. In the last few years, the phenomenon of charge ordering has also played an 
important part in improving and even expanding the structure of the phase diagram of $(\mathrm{TM})_{2} \mathrm{X}$. Though its observation has been so far restricted to members of the Fabre salts series, this phenomenon raises important questions about the influence of charge disproportionation in the relative stability of the spin-Peierls and Néel states in this series of compounds.

The interplay of different types of commensurability in weakly dimerized quarter-filled compounds like the $(\mathrm{TM})_{2} \mathrm{X}$, is another issue that is at the heart of a better understanding of strong electronic correlations that characterize the properties normal phase in $(\mathrm{TM})_{2} \mathrm{X}$. This problem is linked to the persistent issue of the dimensionality crossover or about how the restoration of a Fermi liquid is achieved in quasi-one-dimensional conductors like the $(\mathrm{TM})_{2} \mathrm{X}$.

As the starting point of the study of the Bechgaard salts more than twenty-five years ago, superconductivity is certainly one of the hardest part of the phase diagram to both explain and characterize. In spite of the recent experimental advances, which again confirm the non conventional nature of superconductivity in $(\mathrm{TM})_{2} \mathrm{X}$, the problem of the symmetry of the supercon-

ducting order parameter, as well as the issue of the presence and location of nodes for the gap, are all enduring questions for which a consensus of views has yet to be reached. Given the experimental constraints and difficulties tied to the use of extremely low temperature and high pressure conditions in $(\mathrm{TM})_{2} \mathrm{X}$, these questions will certainly continue to consume major experimental efforts in the next few years.

The mechanism of organic superconductivity in quasi-one-dimensional molecular crystals is a related key issue in want of a satisfactory explanation. The extensive experimental evidence in favor of the systematic emergence of superconductivity in $(\mathrm{TM})_{2} \mathrm{X}$ just below their stability threshold for antiferromagnetism has shown the need for a unified description of electronic excitations that at the core of both density-wave and superconducting correlations. In this matter, the recent progress achieved by the renormalization group method have resulted in definite predictions about the possible symmetries of the superconducting order parameter when a purely electronic mechanism is involved - predictions that often differ from phenomenologically based approaches to superconductivity. The results for the electron gas model, albeit appealing when confronted to existing data, remain only indicative, however, of what may be the actual origin of superconductivity in these complex materials. In this respect, the future progress on the experimental side will be certainly decisive for the theory.

\section{Acknowledgments}

A large part of the results presented in this review are based on a long term activity in the domain of low dimensional organic superconductors with a large number of contributors at Orsay and Sherbrooke. We acknowledge in particular the recent contributions of P. Auban-Senzier, J. C. Nickel, R. 
Duprat and N. Dupuis. This extended activity would not have been possible without the fruitful cooperation of our colleagues in Chemistry J. M. Fabre (Montpellier), K. Bechgaard (Copenhagen), and P. Batail (Angers).

\section{References}

1. K. Bechgaard, D. O. Cowan, and A. N. Bloch, Journal of the Chemical Society Chemical Communications 22, 937 (1974).

2. A. Garito and A. Heeger, Accounts of Chemical Research 7, 232 (1974).

3. E. Engler et al., Jour. Am. Chem. Soc. 99, 5909 (1977).

4. J. Andersen et al., Acta Cryst. B 34, 1901 (1978).

5. A. Andrieux et al., J. Physique. Paris 40, 1199 (1979).

6. G. Brun et al., C.R. Acad. Sc. (Paris) 284 C, 211 (1977).

7. C. Coulon et al., J. Phys. (Paris) 43, 1059 (1982).

8. L. Ducasse et al., J. Phys. C 19, 3805 (1986).

9. K. Behnia et al., Phys. Rev. Lett. 74, 5272 (1995).

10. D. Jérome, Science 252, 1509 (1991).

11. P. Auban, D. Jérome, C. Carcel, and J. Fabre, J. Phys. IV (France) 114, 41 (2004), and to be published.

12. D. Jaccard et al., J. Phys.: Cond. Matt. 13, 89 (2001).

13. Y. A. Bychkov, L. P. Gorkov, and I. Dzyaloshinskii, Sov. Phys. JETP 23, 489 (1966).

14. I. E. Dzyaloshinskii and A. I. Larkin, Sov. Phys. JETP 34, 422 (1972).

15. J. Solyom, Adv. Phys. 28, 201 (1979).

16. S. Barisic and S. Brazovskii, in Recent Developments in Condensed Matter Physics, edited by J. T. Devreese (Plenum, New York, 1981), Vol. 1, p. 327.

17. V. J. Emery, R. Bruisma, and S. Barisic, Phys. Rev. Lett. 48, 1039 (1982).

18. C. Bourbonnais, J. Phys. I (France) 3, 143 (1993).

19. M. Kimura, Prog. Theor. Phys. 63, 955 (1975).

20. T. Giamarchi, Quantum Physics in One Dimension (Oxford University Press, Oxford, 2004).

21. J. Voit, Rep. Prog. Phys. 58, 977 (1995).

22. H. J. Schulz, G. Cuniberti, and P. Pieri, cond-mat/9807366 (unpublished).

23. T. Giamarchi, Physica B230-232, 975 (1997).

24. F. Mila and X. Zotos, Europhys. Lett. 24, 133 (1993).

25. H. J. Schulz, in Strongly Correlated Electronic Materials: The Los Alamos Symposium 1993, edited by K. S. Bedell et al. (Addison-Wesley, Reading, MA, 1994), p. 187.

26. M. Tsuchiizu, H. Yoshioka, and Y. Suzumura, J. Phys. Soc. Jpn. 70, 1460 (2001).

27. C. Bourbonnais et al., J. Phys. (Paris) Lett. 45, L755 (1984).

28. C. Bourbonnais and L. G. Caron, Physica 143B, 450 (1986).

29. S. Brazovskii and Y. Yakovenko, Sov. Phys. JETP 62, 1340 (1985).

30. C. Bourbonnais and L. G. Caron, Int. J. Mod. Phys. B 5, 1033 (1991).

31. C. Bourbonnais and L. G. Caron, Europhys. Lett. 5, 209 (1988).

32. C. Bourbonnais, in Les Houches, Session LVI (1991), Strongly interacting fermions and high- $T_{c}$ superconductivity, edited by B. Doucot and J. ZinnJustin (Elsevier Science, Amsterdam, 1995), p. 307. 
33. C. Bourbonnais, in Trends in High Magnetic Fields Science, edited by L. P. L. C. Berthier, P. Boebinger and G. Martinez (Springer, Heidelberg, 2002), arXiv. cond-mat/0204345.

34. B. Horovitz, H. Gutfreund, and M. Weger, Mol. Cryst. Liq. Cryst. 79, 591 (1982).

35. K. Yamaji, J. Phys. Soc. of Japan 51, 2787 (1982).

36. J. R. Cooper, Phys. Rev. B 19, 2404 (1979).

37. D. Jérome, in Organic Conductors: fundamentals and applications, edited by J.-P. Farges (Dekker, New York, 1994), pp. 405-494.

38. P. Auban-Senzier, D. Jérome, and J. Moser, in Physical Phenomena at High Magnetic Fields, edited by R. S. Z. Fisk, L. P. Gorkov (World Scientific, Singapore, 1999), p. 211.

39. B. Gallois, Ph.D. thesis, Univ. Bordeaux I, 1987.

40. S. Brazovskii and S. A. Gordyunin, JETP Lett. 31, 371 (1980).

41. F. Creuzet et al., Synthetic Metals 19, 277 (1987).

42. P. Wzietek et al., J. Phys. I 3, 171 (1993).

43. N. Miljak, J. R. Cooper, and K. Bechgaard, J. Phys. Coll. 44, 893 (1983).

44. N. Miljak and J. R. Cooper, Mol. Cryst. Liq. Cryst. 119, 141 (1985).

45. N. Mott, Metal-Insulator Transitions (Taylor and Francis, London, 1974).

46. P. Auban-Senzier (unpublished).

47. J. Moser et al., Eur. Phys. J. B 1, 39 (1998).

48. A. Georges, T. Giamarchi, and N. Sandler, Phys. Rev. B 61, 16393 (2000).

49. C. Jacobsen, D. Tanner, and K. Bechgaard, Phys. Rev. B 28, 7019 (1983).

50. M. Dressel, Naturwissenschaften 90, 337 (2003).

51. C. Bourbonnais et al., Phys. Rev. Lett. 62, 1532 (1989).

52. F. Creuzet et al., Synthetic Metals 19, 289 (1987).

53. B. Gotschy et al., J. Phys. I (France) 2, 677 (1992).

54. J. Pouget et al., Mol. Cryst. Liq. Cryst. 79, 129 (1982).

55. P. Foury-Leylekian et al., Phys. Rev. B 70, 180405(R) (2004).

56. M. Dumm et al., Phys. Rev. B 61, 511 (2000).

57. C. Bourbonnais and B. Dumoulin, J. Phys. I (France) 6, 1727 (1996).

58. L. G. Caron, C. Bourbonnais, F. Creuzet, and D. Jerome, Synthetic Metals 19, 69 (1987).

59. J. Riera and D. Poilblanc, Phys. Rev. B 62, R16243 (2000).

60. J. P. Pouget, in Low Dimensional Conductors and Superconductors, edited by D. Jérome and L. G. Caron (Plenum Press, New York, 1987), p. 17.

61. D. Chow et al., Phys. Rev. Lett. 81, 3984 (1998).

62. F. Zamborszky et al., Phys. Rev. B 66, 081103(R) (2002).

63. V. Ilakovac et al., Phys. Rev. B 50, 7136 (1994).

64. D. S. Chow et al., Phys. Rev. Lett. 85, 1698 (2000).

65. L. G. Caron et al., Synthetic Metals 27B, 123 (1988).

66. J. P. Pouget et al., Phys. Rev. Lett. 37, 437 (1976).

67. L. G. Caron and C. Bourbonnais, Phys. Rev. B 29, 4230 (1984).

68. L. G. Caron and S. Moukouri, Phys. Rev. Lett 76, 4050 (1996).

69. H. Bakrim and C. Bourbonnais (unpublished).

70. For a more details about the charge ordered state in the (TMTTF) ${ }_{2} \mathrm{X}$ series, see the contributions of P. Monceau et al.; S. B. Brown and P. M. Chaikin; and S. Brazovskii.

71. F. Nad, P. Monceau, C. Carcel, and J. M. Fabre, Phys. Rev. B 62, 1753 (2000). 
72. F. Nad, P. Monceau, C. Carcel, and J. M. Fabre, J. Phys.: Condens. Matter 12, L435 (2000).

73. P. Monceau, F. Nad, and S. Brazovskii, Phys. Rev. Lett 86, 4080 (2001).

74. C. Coulon, S. S. P. Parkin, and R. Laversanne, Phys. Rev. B 31, 3583 (1985).

75. R. Laversanne et al., J. Phys. (Paris) Lett. 45, L393 (1984).

76. W. Yu et al., Phys. Rev. B 70, R121101 (2004).

77. H. Yoshioka, M. Tsuchiizu, and Y. Suzumura, J. Phys. Soc. Jpn. 70, 762 (2001).

78. J. Riera and D. Poilblanc, Phys. Rev. B 63, R241102 (2001).

79. H. Seo and H. Fukuyama, J. Phys. Soc. Jpn. 66, 1249 (1997).

80. R. T. Clay, S. Mazumdar, and D. K. Campbell, Phys. Rev. B 67, 115121 (2003).

81. J. P. Pouget and S. Ravy, Synthetic Metals 85, 1523 (1997).

82. B. J. Klemme et al., Phys. Rev. Lett. 75, 2408 (1995).

83. S. E. Brown et al., Synthetic Metals 86, 1937 (1997).

84. P. Auban et al., J. Phys. (France) 50, 2727 (1989).

85. P. Auban, D. Jérome, I. Johannsen, and K. Bechgaard, Synthetic Metals 41, 2285 (1991).

86. C. Bourbonnais and D. Jérome, Science 281, 1156 (1998).

87. K. Bechgaard et al., Solid State Comm. 33, 1119 (1980).

88. S. Tomic et al., J. Phys. I (France) 1, 1603 (1991).

89. C. Bourbonnais and D. Jérome, in Advances in Synthetic Metals, Twenty Years of Progress in Science and Technology, edited by P. Bernier, S. Lefrant, and G. Bidan (Elsevier, New York, 1999), pp. 206-261, arXiv:condmat/9903101.

90. A. Schwartz et al., Phys. Rev. B 58, 1261 (1998).

91. C. Jacobsen, in Low Dimensional Conductors and Superconductors, edited by D. Jérome and L. Caron (Plenum Press, New York, 1987), p. 253.

92. T. Timusk, in Low Dimensional Conductors and Superconductors, edited by D. Jérome and L. Caron (Plenum Press, New York, 1987), p. 275.

93. K. Kikuchi et al., Solid State Comm. 42, 433 (1982).

94. K. Bechgaard, M. Carneiro, M. Olsen, and F. Rasmussen, Phys. Rev. Lett. 46, 852 (1981).

95. H. Ng, T. Timusk, D. Jérome, and K. Bechgaard, Phys. Rev. B. 32, 8041 (1985).

96. H. Ng, T. Timusk, and K. Bechgaard, J. Physique 44, C3 (1983).

97. N. Joo et al., Eur. Phys. J. B 40, 43 (2004).

98. K. Heuzé et al., Adv. Materials 15, 1251 (2003).

99. T. Itou et al., Phys. Rev. Lett. 93, 216408 (2004).

100. E. B. Lopes et al., Eur. Phys. J. B 29, 27 (2002).

101. D. Jérome, A. Mazaud, M. Ribault, and K. Bechgaard, J. Phys. (Paris) Lett. 41, L95 (1980).

102. R. Brusetti, M. Ribault, D. Jerome, and K.Bechgaard, J. Phys. (Paris) 43, 801 (1982).

103. K. Mortensen, Y. Tomkiewiez, T. D. Schultz, and E. M. Engler, Phys. Rev. Lett. 46, 1234 (1982).

104. A. Andrieux, D. Jérome, and K. Bechgaard, J. Phys. (Paris) Lett. 42, L87 (1981).

105. T. Adachi et al., J. Am. Chem. Soc. 122, 3238 (2000). 
106. L. Balicas et al., J. Phys. I (France) 4, 1539 (1994).

107. P. Auban-Senzier et al., Synthetic Metals 133-134, 11 (2003).

108. C. S. Jacobsen, D. Tanner, and K. Bechgaard, Phys. Rev. Lett. 46, 1142 (1981).

109. V. Vescoli et al., Science 281, 1181 (1998).

110. T. Takahashi, Y. Maniwa, H. Kawamura, and G. Saito, Physica 143B, 417 (1986).

111. G. Montambaux, Phys. Rev. B 38, 4788 (1988).

112. L. Gor'kov, J. Phys. I (France) 6, 1697 (1996).

113. C. Seidel and V. N. Prigodin, J. Phys. (Paris) Lett. 44, L403 (1983).

114. R. Duprat and C. Bourbonnais, Eur. Phys. J. B 21, 219 (2001).

115. D. Jérome and H. Schulz, Adv. in Physics 31, 299 (1982).

116. T. Vuletic et al., Eur. Phys. J. B 25, 319 (2002).

117. Y. Hasegawa and H. Fukuyama, J. Phys. Soc. Jpn. 55, 3978 (1986).

118. J. F. Kwak et al., Phys. Rev. Lett. 56, 972 (1986).

119. J. P. Pouget and S. Ravy, J. Phys. I (France) 6, 1501 (1996).

120. S. Kagoshima et al., Solid State Comm. 110, 479 (1999).

121. H. K. Ng, T. Timusk, and K. Bechgaard, Phys. Rev. B 30, 5842 (1984).

122. N. Cao, T. Timusk, and K. Bechgaard, J. Phys. I (France) 6, 1719 (1996).

123. S. Mazumdar, R. Ramasesha, R. T. Clay, and D. K. Campbell, Phys. Rev. Lett. 82, 1522 (1999).

124. M. Kobayashi, M. Ogata, and K. Yonemitsu, J. Phys. Soc. Jpn. 67, 1098 (1998).

125. J. C. Nickel, R. Duprat, C. Bourbonnais, and N. Dupuis, Phys. Rev. Lett. 95, 247001 (2005).

126. M. Ribault, G. Benedek, D. Jérome, and K. Bechgaard, J. Phys. Lett. Paris 41, L (1980).

127. K. Andres et al., Phys. Rev. Lett. 45, 1449 (1980).

128. P. Garoche, R. Brusetti, and K. Bechgaard, Phys. Rev. Lett. 49, 1346 (1982).

129. R. Brusetti, P. Garoche, and K. Bechgaard, J. Phys. C 16, 2495 (1983).

130. S. Belin and K. Behnia, Phys. Rev. Lett. 79, 2125 (1997).

131. J. Bardeen, G. Rickaysen, and L. Teword, Phys. Rev. 113, 982 (1959).

132. M. Takigawa, H. Yasuoka, and G. Saito, J. Phys. Soc. Jpn 56, 873 (1987).

133. Y. Hasegawa and H. Fukuyama, J. Phys. Soc. Jpn. 56, 877 (1987).

134. I. J. Lee et al., Phys. Rev. Lett. 88, 17004 (2002).

135. L. P. Le et al., Phys.Rev.B. 48, 7284 (1993).

136. G. M.Luke et al., Physica B326, 378 (2003).

137. G. M. Luke et al., Nature 394, 558 (1998).

138. D. U. Gubser et al., Mol. Cryst. Liq. Cryst. 79, 225 (1982).

139. R. L. Greene et al., Mol. Cryst. Liq. Cryst. 79, 183 (1982).

140. K. Murata et al., Mol. Cryst. Liq. Cryst. 79, 283 (1982).

141. K. Murata et al., Japanese Journal of Applied Physics 26, 1367 (1987).

142. N. Joo, to be published (2006).

143. L. P. Gorkov and D. Jérome, J. Phys. Lett. 46, L643 (1985).

144. I. J. Lee, P. M. Chaikin, and M. J. Naughton, Phys. Rev. B 62, R14669 (2000).

145. J. I. Oh and M. J. Naughton, Phys. Rev. Lett. 92, 067001 (2004).

146. H. Wilhelm (2004), unpublished data and private communication.

147. H. J. Schulz et al., J. Physique. Lett 42, L (1981).

148. L. Jennings and C. Swenson, Phys. Rev 112, 31 (1958). 
149. D. Mailly, Ph.D. thesis, Thesis Université d'Orsay, 1983.

150. F. Creuzet, D. Jérome, and A. Moradpour, Mol. Cryst. Liq. Cryst. 119, 297 (1985).

151. K. Murata et al., Mol. Cryst. Liq. Cryst. 119, 245 (1985).

152. F. Guo et al., J. Phys. Soc. Jpn. 67, 3000 (1998).

153. W. Kang, S. Hannahs, and P. Chaikin, Phys. Rev. Lett. 70, 3091 (1993).

154. I. J. Lee et al., Phys. Rev. Lett. 94, 197001 (2005).

155. R. L. Greene and E. M. Engler, Phys. Rev. Lett. 45, 1587 (1980).

156. M. Héritier, G. Montambaux, and P. Lederer, J. Phys. (Paris) Lett. 45, L943 (1984).

157. L. P. Gorkov and P. D. Grigoriev, Europhys. Lett. 71, 425 (2005).

158. P. Auban-Senzier, (2006), to be published.

159. C. Colin and P. Auban-Senzier and C. R. Pasquier and K. Bechgaard, preprint (2006).

160. S. Bouffard et al., J. Phys. C 15, 295 (1982).

161. M.-Y. Choi et al., Phys. Rev. B 25, 6208 (1982).

162. A. A. Abrikosov, J. Low Temp. Phys 53, 359 (1983).

163. L. Zuppirolli, in Low Dimensional Conductors and Superconductors, edited by D. Jérome and L. G. Caron (Plenum Press, New York, 1987), p. 307.

164. M. Sanquer and S. Bouffard, Mol. Cryst. Liq. Cryst. 119, 147 (1985).

165. I. Johannsen et al., Mol. Cryst. Liq. Cryst. 119, 277 (1985).

166. K. Mortensen and E. M. Engler, Phys. Rev. B 29, 842 (1984).

167. S. Tomic, Jérome, P. Monod, and K. Bechgaard, J. de Phys. (Paris) Lett. 43, L839 (1982).

168. B. Korin-Hamzić, L. Forró, J. R. Cooper, and K. Bechgaard, Phys. Rev. B 38, 11177 (1988).

169. S. Ravy, R. Moret, J. P. Pouget, and R. Comès, Physica 143 B, 542 (1986).

170. N. Joo et al., Europhys. Lett. 72, 645 (2005).

171. K. Maki, H. Won, and S. Haas, Phys. Rev. B 69, 012502 (2004).

172. A. I. Larkin, Sov. Phys. JETP 21, 153 (1965).

173. A. G. Lebed, JETP Lett. 44, 114 (1986).

174. N. Dupuis, G. Montambaux, and C. A. R. S. de Melo, Phys. Rev. Lett. 70, 2613 (1993).

175. The claim for triplet superconducting pairing in $(\mathrm{TM})_{2} \mathrm{X}$ is based essentially on NMR investigations $\left({ }^{77} \mathrm{Se}\right.$ Knight shift and spin-lattice relaxation time $\left.T_{1}\right)$ performed on (TMTSF) ${ }_{2} \mathrm{PF}_{6}$ under pressure [134]. A major difficulty regarding this experiment is the actual control of the temperature of the electron spins during the time of the NMR pulse and acquisition. It is not unlikely that the electron spins can reach a temperature above $T_{c}$ for the experiment leading to the determination of the Knight shift, see also recent data obtained by Shinagawa et-al on (TMTSF) ${ }_{2} \mathrm{ClO}_{4}$ (Proceedings of ISCOM 05).

176. H. Mayaffre et al., Phys. Rev. Lett 75, 4951 (1995).

177. K. Kanoda, K. Miyagawa, A. Kawamoto, and Y. Nakazawa, Phys. Rev. B. 54, 76 (1996).

178. S. Kamiya et al., Phys. Rev. B 65, 134510 (2002).

179. F. Zuo et al., Phys. Rev. B . 61, 750 (2000).

180. D. Jérome and C. R. Pasquier, in One Dimensional Organic Superconductors, edited by A. V. Narlikar (Springer Verlag, Berlin, 2005).

181. N. Dupuis, C. Bourbonnais, and J. C. Nickel, Fiz. Nizk. Temp. 32, 505 (2006), arXiv:cond-mat/0510544. 
182. V. J. Emery, J. Phys. (Paris) Coll. 44, 977 (1983).

183. W. Kang, S. T. Hannahs, and P. M. Chaikin, Phys. Rev. Lett. 70, 3091 (1993).

184. T. Takahashi, Jérome, and K. Bechgaard, J. de Phys. (Paris) Lett. 43, L565 (1982).

185. H. Schwenk, K. Andres, and F. Wudl, Phys. Rev. B 29, 500 (1984).

186. W. Wu et al., Phys. Rev. Lett. 94, 097004 (2005).

187. W. Kohn and J. M. Luttinger, Phys. Rev. Lett. 15, 524 (1965).

188. V. J. Emery, Synthetic Metals 13, 21 (1986).

189. M. T. Béal-Monod, C. Bourbonnais, and V. J. Emery, Phys. Rev. B 34, 7716 (1986).

190. L. G. Caron and C. Bourbonnais, Physica 143B, 453 (1986).

191. D. J. Scalapino, E. Loh, and J. E. Hirsch, Phys. Rev. B 34, R8190 (1986).

192. H. Shimahara, J. Phys. Soc. Jpn. 58, 1735 (1989).

193. H. Kino and H. Kontani, J. Phys. Soc. Jpn. 68, 1481 (1999).

194. J. C. Nickel, R. Duprat, C. Bourbonnais, and N. Dupuis, Phys. Rev. B 73, 094616 (2006).

195. C. Bourbonnais and R. Duprat, J. de Phys. IV 114, 3 (2004).

196. Y. Fuseya and Y. Suzumura, J. Phys. Soc. Jpn. 74, 1263 (2005).

197. A. G. Lebed, K. Machida, and M. Ozaki, Phys. Rev. B 62, R795 (2000).

198. K. Kuroki, R. Arita, and H. Aoki, Phys. Rev. B 63, 094509 (2001).

199. J. P. Pouget and R. Comes, in Charge Density Waves in Solids, edited by L. P. Gor'kov and G. Gruner (Elsevier Science, Amsterdam, 1989), p. 85.

200. S. Barisic and A. Bjelis, in Theoretical Aspects of Band Structures and Electronic Properties of Pseudo-One-Dimensional Solids, edited by H. Kaminura (D. Reidel, Dordrecht, 1985), p. 49.

201. L. P. Gor'kov and I. E. Dzyaloshinskii, Sov. Phys. JETP 40, 198 (1974).

202. P. A. Lee, T. M. Rice, and R. A. Klemm, Phys. Rev. B 15, 2984 (1977). 